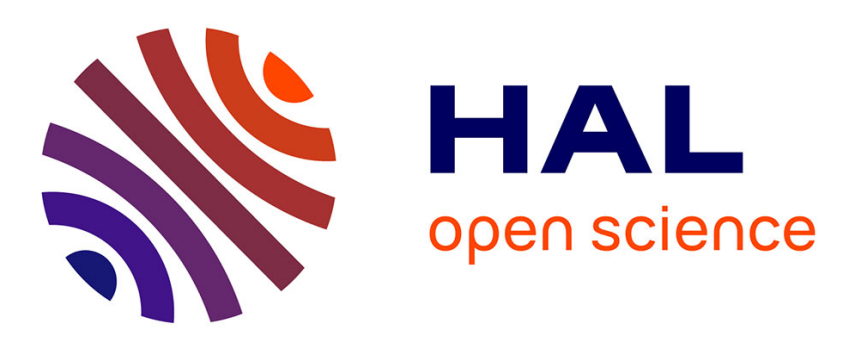

\title{
The analysis of exact controllability of neutral type systems by the moment problem approach
}

\author{
Rabah Rabah, Grigory M. Sklyar
}

\section{To cite this version:}

Rabah Rabah, Grigory M. Sklyar. The analysis of exact controllability of neutral type systems by the moment problem approach. SIAM Journal on Control and Optimization, 2007, 46 (6), pp.2148-2181. 10.1137/060650246. hal-00580060

\section{HAL Id: hal-00580060 https://hal.science/hal-00580060}

Submitted on 25 Mar 2011

HAL is a multi-disciplinary open access archive for the deposit and dissemination of scientific research documents, whether they are published or not. The documents may come from teaching and research institutions in France or abroad, or from public or private research centers.
L'archive ouverte pluridisciplinaire HAL, est destinée au dépôt et à la diffusion de documents scientifiques de niveau recherche, publiés ou non, émanant des établissements d'enseignement et de recherche français ou étrangers, des laboratoires publics ou privés. 


\title{
THE ANALYSIS OF EXACT CONTROLLABILITY OF NEUTRAL-TYPE SYSTEMS BY THE MOMENT PROBLEM APPROACH*
}

\author{
RABAH RABAH ${ }^{\dagger}$ AND GRIGORY M. SKLYAR ${ }^{\ddagger}$
}

\begin{abstract}
The problem of exact null-controllability is considered for a wide class of linear neutral-type systems with distributed delay. The main tool of the analysis is the application of the moment problem approach and the theory of the basis property of exponential families. A complete characterization of this problem is given. The minimal time of controllability is specified. The results are based on the analysis of the Riesz basis property of eigenspaces of the neutral-type systems in Hilbert space.
\end{abstract}

Key words. neutral-type systems, exact controllability, moment problem, Riesz basis, distributed delays

AMS subject classifications. 93B05, 93C23, 93C25

DOI. $10.1137 / 060650246$

1. Introduction. Many applied problems from physics, mechanics, biology, and other fields can be described by partial differential equations or delay differential equations. This leads to the construction and study of the infinite-dimensional system theory concerning also the systems with control. In this context the problem of controllability for distributed parameter systems leads to the study of the abstract controllability problem in infinite-dimensional spaces, which may be formulated in Hilbert spaces as follows. Consider the abstract system

$$
\dot{x}=\mathcal{A} x+\mathcal{B} u,
$$

where $x(t) \in X, u(t) \in U, X$ and $U$ being Hilbert spaces, $\mathcal{A}$ is the generator of a $C_{0^{-}}$ semigroup $\mathrm{e}^{\mathcal{A} t}$, and $\mathcal{B} \in \mathcal{L}(U, X)$ is a bounded operator. The problem of controllability is to find all the states $x_{T}$ that can be reached from a fixed initial state (say 0 ) at a finite time $T$ by the choice of the controls $u(\cdot) \in L_{2}(0, T ; U)$. The mild solution of the system (1.1) is given by

$$
x(t)=\mathrm{e}^{\mathcal{A} t} x(0)+\int_{0}^{t} \mathrm{e}^{\mathcal{A}(t-\tau)} \mathcal{B} u(\tau) \mathrm{d} \tau .
$$

The reachability set from 0 at time $T$ is defined by

$$
\mathcal{R}_{T}=\left\{x: x=\int_{0}^{T} \mathrm{e}^{\mathcal{A} t} \mathcal{B} u(t) \mathrm{d} t, \quad u(\cdot) \in L_{2}(0, T ; U)\right\} .
$$

* Received by the editors January 18, 2006; accepted for publication (in revised form) June 11, 2007; published electronically December 21, 2007. This work was partially supported by the FrenchPolish grant Polonium 07599VH, by École Centrale de Nantes, and by the French research center CNRS.

http://www.siam.org/journals/sicon/46-6/65024.html

${ }^{\dagger}$ IRCCyN, UMR CNRS 6597, École des Mines de Nantes, 4 rue Alfred Kastler, BP 2072244307 Nantes Cedex 3, France (rabah@emn.fr).

${ }^{\ddagger}$ Institute of Mathematics, University of Szczecin, Wielkopolska 15, 70451 Szczecin, Poland (sklar@univ.szczecin.pl). 
For finite-dimensional systems the natural concept of controllability is when $\mathcal{R}_{T}=$ $X$ (Kalman). For infinite-dimensional systems, as has been pointed out by several authors (Fattorini, Triggiani, Russel, Balakrishnan, and others), this concept is not realistic. It is easy to show that $\mathcal{R}_{T_{1}} \subset \mathcal{R}_{T_{2}}$ as $T_{1}<T_{2}$. In general, there is no universal time $T_{0}$ such that $\mathcal{R}_{T_{0}}=\mathcal{R}_{T}$ for all $T>T_{0}$. However, for several classes of systems important for application this property holds (hyperbolic-type and neutraltype systems). In these cases, a natural way to formulate the controllability problem is the following setting:

(i) to find the maximal possible set $\mathcal{R}_{T}$ (depending on $T$ ),

(ii) to find the minimal $T$ for which the set $\mathcal{R}_{T}$ becomes the maximal possible.

In order to obtain more profound and precise results by using this approach, it is important to consider a concrete class of systems and to use the specificity of this class. In this paper, we consider the problem of controllability for a general class of neutral systems with distributed delays given by the equation

$$
\left\{\begin{array}{l}
\frac{\mathrm{d}}{\mathrm{d} t}\left[z(t)-K z_{t}\right]=L z_{t}+B u(t), \quad t \geq 0 \\
z_{0}=f
\end{array}\right.
$$

where $z_{t}:[-1,0] \rightarrow \mathbb{C}^{n}$ is the history of $z$ defined by $z_{t}(s)=z(t+s)$. The difference and delay operators $K$ and $L$, respectively, are defined by

$$
K f=A_{-1} f(-1) \quad \text { and } \quad L f=\int_{-1}^{0} A_{2}(\theta) \frac{\mathrm{d}}{\mathrm{d} \theta} f(\theta) \mathrm{d} \theta+\int_{-1}^{0} A_{3}(\theta) f(\theta) \mathrm{d} \theta
$$

for $f \in H^{1}\left([-1,0], \mathbb{C}^{n}\right)$, where $A_{-1}$ is a constant $n \times n$ matrix, $A_{2}, A_{3}$ are $n \times n$ matrices whose elements belong to $L_{2}(-1,0)$, and $B$ is a constant $n \times r$ matrix.

We consider the operator model of the neutral-type system (1.2) introduced by Burns, Herdman, and Stech [3] in product spaces (see also [5]). The state space is $M_{2}\left(-1,0 ; \mathbb{C}^{n}\right)=\mathbb{C}^{n} \times L_{2}\left(-1,0 ; \mathbb{C}^{n}\right)$, shortly $M_{2}$, and $(1.2)$ can be reformulated as

$$
\dot{x}(t)=\mathcal{A} x(t)+\mathcal{B} u(t), \quad x(0)=\left(\begin{array}{c}
y \\
f
\end{array}\right), \quad \mathcal{B}=\left(\begin{array}{c}
B \\
0
\end{array}\right), \quad \mathcal{A}=\left(\begin{array}{cc}
0 & L \\
0 & \frac{\mathrm{d}}{\mathrm{d} \theta}
\end{array}\right),
$$

with $\mathcal{D}(\mathcal{A})=\left\{(y, z(\cdot)) \in M_{2}: z \in H^{1}\left([-1,0] ; \mathbb{C}^{n}\right), y=z(0)-A_{-1} z(-1)\right\}$.

In the particular case when $A_{2}(\theta)=A_{3}(\theta)=0$, which corresponds to $L=0$, we will use the notation $\widetilde{\mathcal{A}}$ for $\mathcal{A}$.

Suppose that the initial condition for the system $(1.2)$ is $z(t)=z_{0}(t), t \in[-1,0]$, and let us put $z_{t}(\theta)=z(t+\theta), \theta \in[-1,0]$, and $y=z(0)-A_{-1} z(-1)$. The semigroup generated by $\mathcal{A}$ is given by

$$
\mathrm{e}^{\mathcal{A} t}\left(\begin{array}{c}
y \\
z_{0}(\cdot)
\end{array}\right)=\left(\begin{array}{c}
z_{t}(0)-A_{-1} z_{t}(-1) \\
z_{t}(\cdot)
\end{array}\right)=\left(\begin{array}{c}
z(t)-A_{-1} z(t-1) \\
z(t+\cdot)
\end{array}\right) .
$$

It can be shown that the reachability set $\mathcal{R}_{T}$ is such that $\mathcal{R}_{T} \subset \mathcal{D}(\mathcal{A})$ for all $T>0$. This is a consequence of the fact that for all $u(\cdot) \in L_{2}$ the corresponding solution of $(1.2)$ is in $H^{1}$ and then the solution of $(1.3)$ is in $\mathcal{D}(\mathcal{A})$ (see [5, Proposition 2.2] for the existence of the solution and [5, Corollary 2.7] for the property of the reachability subset). This naturally leads to the following definition of exact controllability.

DEFINITION 1.1. The system (1.3) is exactly null-controllable by controls from $L_{2}$ at the time $T$ if $\mathcal{R}_{T}=\mathcal{D}(\mathcal{A})$. This means that the set of solutions of the system (1.2), $\{z(t), t \in[T-1, T]\}$, coincides with $H^{1}\left([T-1, T] ; \mathbb{C}^{n}\right)$. 
This problem was the focus of attention of several authors in the 1970s and 1980s. The main results were devoted to systems with one or several discrete delays. This may be explained by the fact that the explicit, in this case, form of solutions is known and, as a result, the semigroup describing the solutions of (1.2) is known explicitly.

The main result for the system

$$
\dot{z}(t)-A_{-1} \dot{z}(t-h)=A_{0} z(t)+A_{1} z(t-h)+B u
$$

is that the exact controllability holds if and only if (see $[9,12])$

(i) $\operatorname{rank}(\Delta(\lambda) \quad B)=n$,

(ii) $\operatorname{rank}\left(\begin{array}{llll}B & A_{-1} B & \cdots & A_{-1}^{n-1} B\end{array}\right)=n$,

where $\Delta(\lambda)=\lambda I-\lambda A_{-1} \mathrm{e}^{-\lambda h}-A_{0}-A_{1} \mathrm{e}^{-\lambda h}$. For the particular case of scalar control ( $B$ is $n \times 1$ matrix) the time of exact controllability is given in [6]: $T>n h$, where $h$ is the delay. For the general case, it is shown in [2] that the reachability set cannot increase for $T>n h$.

The case of noncommensurate delays with a distributed term was precisely studied in the paper by Yamamoto [16]. General conditions were given using the input-output technique. Conditions of approximate controllability (in [16], quasi reachability) in the time domain were explicitly given for a system without distributed delay (see also $[8])$.

In contrast to the above-mentioned works, we consider the model with distributed delays (1.2). In this case, we know only that the solution of (1.2) exists but the corresponding semigroup is not explicitly known. Then the technique using the explicit form of the solution, via an expression of the semigroup, cannot be used. So one needs another tool to analyze the controllability. In the similar situation of the controllability problems for hyperbolic systems, the powerful technique of the moment problem has been proved to be useful. It is caused by the fact that the operators corresponding to hyperbolic systems are as a rule skew-adjoint or close to skew-adjoint and then they possess a basis of eigenvectors. The expansion of the steering conditions in this basis allows the controllability problem for these systems to be reduced to a trigonometric problem with respect to some families of exponentials. Thus, the further analysis concerns the solvability of the non-Fourier trigonometric moment problem and is based on the profound theory of the Riesz bases of exponentials. This theory, originated by the famous Paley-Wiener theorem, has essentially been developed in the last decades (see monographs by Avdonin and Ivanov [1] and by Young [17] and the references therein).

The main idea of our work is to apply the moment problem method to the analysis of controllability of neutral-type systems. Note in this context that the case of neutraltype systems differs essentially from those mentioned above since the operator $\mathcal{A}$ of the system is not skew-adjoint and, moreover, may not have a basis of eigenvectors or even generalized eigenvectors. The first element of our consideration is the spectral analysis of the operator model (1.3) given in our previous works, together with Rezounenko $[10,11]$. In these papers, it is shown that, under the condition that the matrix $A_{-1}$ is not singular, the operator $\mathcal{A}$ (even if it does not verify the Riesz basis property) possesses a Riesz basis of finite-dimensional invariant subspaces. This allows the construction of a special Riesz basis in the space $M_{2}$ in which the steering conditions

$$
\left(\begin{array}{c}
y_{T} \\
z_{T}(\cdot)
\end{array}\right)=\int_{0}^{T} \mathrm{e}^{\mathcal{A}(T-\tau)} \mathcal{B} u(\tau) \mathrm{d} \tau
$$

take the form of a moment problem quadratically close to some special non-Fourier moment problems with respect to a family of quasi polynomials. These questions are 
considered in sections 2 and 3. Let us notice that the detailed attention accorded to the construction of the needed Riesz basis is essentially motivated by the fact that, in the general case, the operator $\mathcal{A}$ may not possess a spectral Riesz basis. Otherwise, for example, if the eigenvalues of the matrix $A_{-1}$ are simple, our construction would be much simpler. The main tool of the analysis of solvability of the obtained moment problem is based on the theory of families of exponentials $[1,17]$. The basic elements of this approach used in our paper are given in section 4 . Below we give a complete analysis of the controllability problem for neutral-type systems. In the course of the main part of the work, we consider the case when $\operatorname{det} A_{-1} \neq 0$. In this case, the controllability of system (1.2) is equivalent to the solvability of the moment problem obtained in section 3. We consider first the single input case in section 5 and give not only the conditions of exact null-controllability but also determine the time of controllability. These results are an extension of the result obtained in [6]. In section 6 , we consider the solvability of the moment problem for the multivariable case ( $\operatorname{dim} B=$ $n \times r, r>1$ ). We introduce some special indices $m_{1}$ and $\bar{m}$ which enable the moment problem to be characterized. We show that the exact null-controllability holds for time $T>\bar{m}$ and does not hold for $T<m_{1}$. Finally, we complete the results on controllability by getting rid of the assumption $\operatorname{det} A_{-1} \neq 0$ in section 7 . We then obtain the precise time of controllability using the first controllability index of the pair $\left(A_{-1}, B\right)$, say $n_{1}$ (cf., for example, [15, Chapter 5]). Our main result is the following.

THEOREM 1.2. The system (1.3) is exactly null-controllable if and only if the following conditions are verified.

(i) There is no $\lambda \in \mathbb{C}$ and $y \in \mathbb{C}^{n}, y \neq 0$, such that $\Delta_{\mathcal{A}}^{*}(\lambda) y=0$ and $B^{*} y=0$, where

$$
\Delta_{\mathcal{A}}^{*}(\lambda)=\lambda I-\lambda \mathrm{e}^{-\lambda} A_{-1}^{*}-\lambda \int_{-1}^{0} \mathrm{e}^{\lambda s} A_{2}^{*}(s) \mathrm{d} s-\int_{-1}^{0} \mathrm{e}^{\lambda s} A_{3}^{*}(s) \mathrm{d} s,
$$

or equivalently $\operatorname{rank}\left(\Delta_{\mathcal{A}}(\lambda) \quad B\right)=n$ for all $\lambda \in \mathbb{C}$.

(ii) There is no $\mu \in \sigma\left(A_{-1}\right)$ and $y \in \mathbb{C}^{n}, y \neq 0$, such that $A_{-1}^{*} y=\bar{\mu} y$ and $B^{*} y=0$, or equivalently $\operatorname{rank}\left(\begin{array}{llll}B & A_{-1} B & \cdots & A_{-1}^{n-1} B\end{array}\right)=n$.

If conditions (i) and (ii) hold, then the system is controllable at the time $T>n_{1}$ and not controllable at the time $T \leq n_{1}$, where $n_{1}$ is the controllability index of the pair $\left(A_{-1}, B\right)$.

If the delay is $h$ instead of 1 , the time of exact controllability is $T=n_{1} h$.

2. The choice of the basis. In this section, we assume that the matrix $A_{-1}$ is not singular, $\operatorname{det} A_{-1} \neq 0$.

Let us recall [10] that the spectrum of $\widetilde{\mathcal{A}}$ (the state operator corresponding to the case $A_{2}=A_{3}=0$ ) consists of only the eigenvalues that are the roots of the equation $\operatorname{det} \Delta_{\widetilde{\mathcal{A}}}(\lambda)=\operatorname{det}\left(\lambda I-\lambda \mathrm{e}^{-\lambda} A_{-1}\right)=0$, i.e.,

$$
\sigma(\widetilde{\mathcal{A}})=\left\{\lambda_{m}^{(k)}=\ln \left|\mu_{m}\right|+\mathrm{i}\left(\arg \mu_{m}+2 k \pi\right)\right\} \cup\{0\},
$$

where $\left\{\mu_{m}, m=1, \ldots, \ell\right\}=\sigma\left(A_{-1}\right)$.

The operator $\widetilde{\mathcal{A}}$ possesses a Riesz basis of generalized eigenvectors which may be characterized as follows (see $[10,11]$ ).

Let $\nu_{m}$ be the number of Jordan blocks corresponding to $\mu_{m} \in \sigma\left(A_{-1}\right)$ and let $p_{m, j}, j=1, \ldots, \nu_{m}$, be the dimension of the corresponding blocks; then 
1. to any $\lambda_{m}^{(k)} \neq 0$ and to any $j=1, \ldots, \nu_{m}$ there corresponds a Jordan chain of generalized eigenvectors of $\widetilde{\mathcal{A}}$, noted $\left\{\widetilde{\varphi}_{m, k}^{j, 1}, \widetilde{\varphi}_{m, k}^{j, 2}, \ldots, \widetilde{\varphi}_{m, k}^{j, p_{m, j}}\right\}$ such that

$$
\widetilde{\mathcal{A}} \widetilde{\varphi}_{m, k}^{j, 1}=\lambda_{m}^{(k)} \widetilde{\varphi}_{m, k}^{j, 1}, \quad\left(\widetilde{\mathcal{A}}-\lambda_{m}^{(k)} I\right) \widetilde{\varphi}_{m, k}^{j, s}=\widetilde{\varphi}_{m, k}^{j, s-1}, \quad s=2, \ldots, p_{m, j} ;
$$

2. the root space of $\widetilde{\mathcal{A}}$ corresponding to $0 \in \sigma(\widetilde{\mathcal{A}})$ is of dimension

$$
n+\operatorname{dim} \operatorname{Ker}\left(A_{-1}-I\right)^{n} .
$$

If $1=\mu_{g} \in \sigma\left(A_{-1}\right), g \in\{1, \ldots, \ell\}$, then for any $j \in\left\{1, \ldots, \nu_{g}\right\}$ there exists a Jordan chain $\left\{\widetilde{\varphi}_{0}^{j, 1}, \widetilde{\varphi}_{0}^{j, 2}, \ldots, \widetilde{\varphi}_{0}^{j, p_{g, j}+1}\right\}$ such that

$$
\widetilde{\mathcal{A}} \widetilde{\varphi}_{0}^{j, 1}=0, \quad \widetilde{\mathcal{A}} \widetilde{\varphi}_{0}^{j, s}=\widetilde{\mathcal{A}} \widetilde{\varphi}_{0}^{j, s-1}, \quad s=2, \ldots, p_{m, j}+1,
$$

and, besides, there exist $n-\nu_{g}$ linearly independent eigenvectors $\widetilde{\varphi}_{0}^{j}, j=$ $\nu_{g}+1, \ldots, n$, such that $\widetilde{\mathcal{A}} \widetilde{\varphi}_{0}^{j}=0$

3. any collection $\left\{\widetilde{\varphi}_{m, k}^{j, s}, s=1, \ldots, p_{m, j}, j=1, \ldots, \nu_{m}\right\}$ forms a basis in the space $\operatorname{Ker}\left(\widetilde{\mathcal{A}}-\lambda_{m}^{(k)} I\right)^{n}, \lambda_{m}^{(k)} \neq 0$. The collection

$$
\left\{\widetilde{\varphi}_{0}^{j, s}, s=1, \ldots, p_{m, j}+1, j=1, \ldots, \nu_{m}\right\} \bigcup\left\{\widetilde{\varphi}_{0}^{j}, j=\nu_{g}+1, n\right\}
$$

forms a basis in $\operatorname{Ker} \widetilde{\mathcal{A}}^{n}$.

In the following, we refer to this basis as $\{\widetilde{\varphi}\}$ omitting the indices when they are not necessary. The concrete form of interest to us is that which corresponds to the nonzero eigenvalues and then takes the form

$$
\widetilde{\varphi}_{m, k}^{j, s}=\left(\begin{array}{c}
0 \\
\mathrm{e}^{\lambda_{m}^{(k)} t} P_{m}^{j, s}(\theta)
\end{array}\right)
$$

with

$$
P_{m}^{j, s}(\theta)=\sum_{r=1}^{s} D_{m, j}^{r} \sum_{i=0}^{s-r} \beta_{r}^{i, s} \frac{\theta^{i}}{i !}
$$

The constant vectors $D_{m, j}^{i}$ form a basis in $\mathbb{C}^{n}$ designed from the Jordan chains of the matrices $A_{-1}$. These vectors and the constants $\beta_{q}^{i, s}$ in the polynomials $P(\theta)$ do not depend on $k$.

This gives that, in particular,

$$
\inf \left\{\left\|\widetilde{\varphi}_{m, k}^{j, s}\right\|, k \in \mathbb{Z}\right\}=\rho>0, \quad \sup \left\{\left\|\widetilde{\varphi}_{m, k}^{j, s}\right\|, k \in \mathbb{Z}\right\}=R<\infty .
$$

The corresponding biorthogonal basis to $\{\widetilde{\varphi}\}$ will be denoted by $\{\widetilde{\psi}\}$.

Proposition 2.1. For any $m, k$ the vectors of the biorthogonal basis $\{\widetilde{\psi}\}$ form the following Jordan chain with respect to the adjoint operator $\widetilde{\mathcal{A}}^{*}$ :

$$
\left(\widetilde{\mathcal{A}}^{*}-\bar{\lambda}_{m}^{(k)} I\right) \widetilde{\psi}_{m, k}^{j, p_{m, j}}=0, \quad\left(\widetilde{\mathcal{A}}^{*}-\bar{\lambda}_{m}^{(k)} I\right) \widetilde{\psi}_{m, k}^{j, s}=\widetilde{\psi}_{m, k}^{j, s+1}, \quad s=0, \ldots, p_{m, j}-1,
$$

where $\bar{\lambda}$ is the complex conjugate of $\lambda$.

Proof. To prove the statement we need to observe that

$$
\left\langle\left(\widetilde{\mathcal{A}}-\lambda_{m}^{(k)} I\right) \widetilde{\varphi}, \widetilde{\psi}_{m, k}^{j, p_{m, j}}\right\rangle=0 \quad \forall \widetilde{\varphi} \in\{\widetilde{\varphi}\} .
$$

Copyright $@$ by SIAM. Unauthorized reproduction of this article is prohibited. 
Hence $\widetilde{\psi}_{m, k}^{j, p_{m, j}} \in \mathcal{D}\left(\widetilde{\mathcal{A}}^{*}\right)$ and $\left(\widetilde{\mathcal{A}}^{*}-\bar{\lambda}_{m}^{(k)} I\right) \widetilde{\psi}_{m, k}^{j, p_{m, j}}=0$. Next, for $s=0, \ldots, p_{m, j}-1$ we have

$$
\left\langle\left(\widetilde{\mathcal{A}}-\widetilde{\lambda}_{m}^{(k)} I\right) \widetilde{\varphi}, \widetilde{\psi}_{m, k}^{j, s}\right\rangle=0 \quad \forall \widetilde{\varphi} \in\{\widetilde{\varphi}\} \backslash\left\{\widetilde{\varphi}_{m, k}^{j, s+1}\right\}
$$

and

$$
\left\langle\left(\widetilde{\mathcal{A}}-\lambda_{m}^{(k)} I\right) \widetilde{\varphi}_{m, k}^{j, s+1}, \widetilde{\psi}_{m, k}^{j, s}\right\rangle=1 .
$$

This means that $\widetilde{\psi}_{m, k}^{j, s} \in \mathcal{D}\left(\widetilde{\mathcal{A}}^{*}\right)$ and $\left(\widetilde{\mathcal{A}}^{*}-\bar{\lambda}_{m}^{(k)} I\right) \widetilde{\psi}_{m, k}^{j, s}=\widetilde{\psi}_{m, k}^{j, s+1}$.

Let us give the concrete form of elements $\widetilde{\psi}_{m, k}^{j, s}$ corresponding to the nonzero eigenvalues.

Proposition 2.2. Let $\left\{C_{m, j}^{1}, \ldots, C_{m, j}^{p_{m, j}}\right\}$ be the $j$ th Jordan chain of $A_{-1}^{*}$ corresponding to $\bar{\mu}_{m}$ :

$$
A_{-1}^{*} C_{m, j}^{1}=\bar{\mu}_{m} C_{m, j}^{1}, \quad\left(A_{-1}^{*}-\bar{\mu}_{m} I\right) C_{m, j}^{s}=C_{m, j}^{s-1}, \quad s=2, \ldots, p_{m, j} .
$$

Then the vectors $\widetilde{\psi}_{m, k}^{j, p_{m, j}-r}$ are of the form

$$
\widetilde{\psi}_{m, k}^{j, p_{m, j}-i}=\left(\begin{array}{c}
\frac{1}{\bar{\lambda}_{m}^{(k)}} \sum_{s=0}^{i} q_{m, j}^{i, 1+s} C_{m, j}^{1+s} \\
\mathrm{e}^{-\bar{\lambda}_{m}^{(k)} \theta} \sum_{s=0}^{i} \widetilde{q}_{m, j}^{i+1+s}(\theta) C_{m, j}^{1+s}
\end{array}\right),
$$

where $i=0, \ldots, p_{m, j}-1$ and the coefficients $q$ and $\widetilde{q}(\theta)$ do not depend on $k$.

In particular, the eigenvectors are given by

$$
\widetilde{\psi}_{m, k}^{j, p_{m . j}}=\beta_{m, k}^{j}\left(\begin{array}{c}
\frac{1}{\bar{\lambda}_{m}^{(k)}} C_{m, j}^{1} \\
\mathrm{e}^{\bar{\lambda}_{m}^{(k)} \theta} C_{m, j}^{1}
\end{array}\right), \quad \beta_{m, k}^{j} \in \mathbb{C} .
$$

Proof. The proof may be obtained by a simple calculation.

Let us now recall [10] that the space $M_{2}$ possesses a Riesz basis of $\mathcal{A}$-invariant finite-dimensional subspaces $\{V\}=\left\{V_{m}^{(k)},|k|>N, m=1, \ldots, \ell\right\} \cup\left\{\widehat{V}_{N}\right\}$, where

$$
V_{m}^{(k)}=P_{m}^{(k)} M_{2}, \quad P_{m}^{(k)}=\frac{1}{2 \pi \mathrm{i}} \int_{L_{m}^{(k)}} R(\lambda, \mathcal{A}) \mathrm{d} \lambda,
$$

where $L_{m}^{(k)}$ are circles of fixed radius $r<r_{0}=\frac{1}{3} \min \left\{\left|\lambda_{m}^{k}-\lambda_{i}^{j}\right|, \quad(m, k) \neq(i, j)\right\}$, centered at $\lambda_{m}^{k}$, and $\widehat{V}_{N}$ is the subspace spanned on all the generalized eigenvectors of $\mathcal{A}$ whose eigenvalues are located outside the circles $L_{m}^{(k)},|k|>N, m=1, \ldots, \ell$, with $\operatorname{dim} \widehat{V}_{N}=2(N+1) n$. Let us remark that this Riesz basis property is valid for all sufficiently large $N \geq N_{0}$. Moreover, it is shown in [10] that

$$
\sum_{k \in \mathbb{Z}} \sum_{m=1}^{\ell}\left\|P_{m}^{(k)}-\widetilde{P}_{m}^{(k)}\right\|^{2}<\infty
$$

where

$$
\widetilde{P}_{m}^{(k)}=\frac{1}{2 \pi \mathrm{i}} \int_{L_{m}^{(k)}} R(\lambda, \widetilde{\mathcal{A}}) \mathrm{d} \lambda, \quad \operatorname{Im} \widetilde{P}_{m}^{(k)}=\operatorname{Ker}\left(\widetilde{\mathcal{A}}-\lambda_{m}^{(k)} I\right)^{n}
$$

Copyright $@$ by SIAM. Unauthorized reproduction of this article is prohibited. 
So, in this sense, the basis $\{V\}$ is asymptotically quadratic close to the spectral basis of $\widetilde{\mathcal{A}}$. Consider now the biorthogonal to $\{V\}$ basis of subspaces

$$
\{W\}=\left\{W_{m}^{(k)},|k|>N, m=1, \ldots, \ell\right\} \cup\left\{\widehat{W}_{N}\right\},
$$

i.e., the basis that may be defined by

$$
W_{m}^{(k)}=\left(\sum_{\substack{(i, j) \neq(m, k) \\|i|>N, j=1, \ldots, \ell}} V_{j}^{(i)}+\widehat{V}_{N}\right)^{\perp}, \quad m=1, \ldots, \ell,|k|>N
$$

and

$$
\widehat{W}_{N}=\left(\sum_{\substack{|i|>N \\ j=1, \ldots, \ell}} V_{j}^{(i)}\right)^{\perp}
$$

One can easily check that the basis $\{W\}$ consists of $\mathcal{A}^{*}$-invariant subspaces and, besides,

$$
W_{m}^{(k)}=P_{m}^{(k) *} M_{2}, \quad P_{m}^{(k) *}=\frac{1}{2 \pi \mathrm{i}} \int_{\bar{L}_{m}^{(k)}} R\left(\lambda, \mathcal{A}^{*}\right) \mathrm{d} \lambda, \quad|k|>N, m=1, \ldots, \ell,
$$

where $\bar{L}_{m}^{(k)}$ are the complex conjugate circles to $L_{m}^{(k)}$.

The finite-dimensional operator $\mathcal{A}_{\mid \widehat{W}_{N}}^{*}$ has the spectrum $\sigma\left(\mathcal{A}_{\mid \widehat{W}_{N}}^{*}\right)$ which is the complex conjugate of the spectrum $\sigma\left(\mathcal{A}_{\mid \widehat{V}_{N}}\right)$. Let us consider this relation in more detail. Let $\widehat{\lambda}_{m}, m=1, \ldots, \ell_{N}$, be the eigenvalues of $\mathcal{A}_{\mid \widehat{V}_{N}}$, and $\widehat{\nu}_{m}$ the number of Jordan blocks corresponding to $\widehat{\lambda}_{m} \in \sigma\left(\mathcal{A}_{\mid \widehat{V}_{N}}\right)$ with the dimension of blocks $\widehat{p}_{m, j}, j=$ $1, \ldots, \widehat{\nu}_{m}$. Let $\widehat{\varphi}_{m}^{j, s}, j=1, \ldots, \widehat{\nu}_{m} ; s=1, \ldots, \widehat{p}_{m, j}$, be a Jordan basis of generalized vectors of $\mathcal{A}_{\mid \widehat{V}_{N}}$, i.e.,

$$
\mathcal{A} \widehat{\varphi}_{m}^{j, 1}=\widehat{\lambda}_{m} \widehat{\varphi}_{m}^{j, 1}, \quad\left(\mathcal{A}-\widehat{\lambda}_{m} I\right) \widehat{\varphi}_{m}^{j, s}=\widehat{\varphi}_{m}^{j, s-1}, \quad s=2, \ldots, \widehat{p}_{m, j},
$$

for the subspace $\widehat{V}_{N}$.

We can now formulate the following statement.

Proposition 2.3. The family

$$
\left\{\widehat{\psi}_{m}^{j, s}, m=1, \ldots, \ell_{N} ; j=1, \ldots, \widehat{\nu}_{m} ; s=1, \ldots, \widehat{p}_{m, j}\right\} \subset \widehat{W}_{N}
$$

biorthogonal to $\left\{\widehat{\varphi}_{m}^{j, s}\right\}$, i.e., $\left\langle\widehat{\varphi}_{m}^{j, s}, \widehat{\psi}_{r}^{i, k}\right\rangle=\delta_{\{(m, j, s),(r, i, k)\}}$, forms a Jordan basis of generalized eigenvectors of $\mathcal{A}_{\mid \widehat{V}_{N}}^{*}$ :

$$
\mathcal{A}^{*} \widehat{\psi}_{m}^{j, \widehat{p}_{m, j}}=\bar{\lambda}_{m} \widehat{\psi}_{m}^{j, \widehat{p}_{m, j}}, \quad\left(\mathcal{A}^{*}-\overline{\widehat{\lambda}}_{m} I\right) \widehat{\psi}_{m}^{j, s}=\widehat{\psi}_{m}^{j, s+1}, \quad s=0, \ldots, \widehat{p}_{m, j}-1,
$$

for the subspace $\widehat{W}_{N}$.

Proof. The proof is analogous to the proof of Proposition 2.1.

Now we have all the elements to define the Riesz basis that we will use for the analysis of the steering condition (1.4).

Copyright (c) by SIAM. Unauthorized reproduction of this article is prohibited. 
We consider the spectral, with respect to the operator $\widetilde{\mathcal{A}}$, basis $\{\widetilde{\varphi}\}$ described above and the corresponding biorthogonal basis $\{\widetilde{\psi}\}$. For a given $N>N_{0}$ we put

$$
\psi_{m, k}^{j, s}=P_{m}^{(k) *} \widetilde{\psi}_{m, k}^{j, s},|k|>N, m=1 \ldots, \ell ; \quad j=1, \ldots, \nu_{m} ; s=1, \ldots, p_{m, j} .
$$

Then we complete the collection (2.8) by the set

$$
\left\{\widehat{\psi}_{m}^{j, s} ; m=1, \ldots, \ell_{N} ; j=1, \ldots, \widehat{\nu}_{m} ; s=1, \ldots, \widehat{p}_{m, j}\right\}
$$

which contains $2(N+1) n$ vectors forming a Jordan basis in $\widehat{W}_{N}$ (Proposition 2.3).

TheOREm 2.4. Let the condition (2.3) be satisfied and let $N$ be sufficiently large. Then the collection

$$
\{\psi\}=\left\{\psi_{m, k}^{j, s}\right\} \cup\left\{\widehat{\psi}_{m}^{j, s}\right\},
$$

where $\psi_{m, k}^{j, s}$ are given by (2.8) and $\psi_{m}^{j, s}$ are defined in Proposition 2.3, constitutes a Riesz basis of $M_{2}$.

Proof. We start with the fact that under condition (2.3) the collection $\{\widetilde{\psi}\}$ forms a Riesz basis in $M_{2}$. In particular, this implies that the collection $\left\{\widetilde{\psi}_{m, k}^{j, s},|k|>N\right\}$ forms a Riesz basis in the closure of its linear span

$$
\mathrm{Cl} \operatorname{Lin}\left\{\widetilde{\psi}_{m, k}^{j, s},|k|>N\right\}=\mathrm{Cl} \sum_{|k|>N} \sum_{m=1}^{\ell} \operatorname{Ker}\left(\widetilde{\mathcal{A}}^{*}-\bar{\lambda}_{m}^{(k)} I\right)^{\max _{j} p_{m, j}} .
$$

On the other hand, from (2.6) and (2.8) we have

$$
\sum_{|k|>N} \sum_{m, j, s}\left\|\psi_{m, k}^{j, s}-\widetilde{\psi}_{m, k}^{j, s}\right\|^{2}=\sum_{|k|>N} \sum_{m, j, s}\left\|P_{m}^{(k) *}-\widetilde{P}_{m}^{(k) *}\right\|^{2}\left\|\widetilde{\psi}_{m, k}^{j, s}\right\|^{2}
$$

This implies that for any $\varepsilon>0$ there exists a large $N_{1}$ such that if $N>N_{1}$, then

$$
\sum_{|k|>N} \sum_{m, j, s}\left\|\psi_{m, k}^{j, s}-\widetilde{\psi}_{m, k}^{j, s}\right\|^{2}<\varepsilon
$$

Hence for this $N$, the family $\left\{\psi_{m, k}^{j, s}\right\}_{|k|>N}$ is quadratically close to $\left\{\widetilde{\psi}_{m, k}^{j, s}\right\}_{|k|>N}$ and, therefore, forms a Riesz basis in the closure of its linear span

$$
\mathrm{Cl} \operatorname{Lin}\left\{\psi_{m, k}^{j, s},|k|>N\right\}=\mathrm{Cl} \sum_{|k|>N} \sum_{m=1}^{\ell} W_{m}^{(k)} .
$$

Since, due to Proposition 2.3, the set $\left\{\widehat{\psi}_{m}^{j, s}\right\}$ is a basis in $\widehat{W}_{N}$ and

$$
\mathrm{Cl} \sum_{|k|>N} \sum_{m}^{\ell} W_{m}^{(k)}+\widehat{W}_{N}=M_{2}
$$

the union

$$
\left\{\psi_{m, k}^{j, s},|k|>N\right\} \cup\left\{\widehat{\psi}_{m}^{j, s}\right\}
$$

Copyright $@$ by SIAM. Unauthorized reproduction of this article is prohibited. 
is a Riesz basis in $M_{2}$. This ends the proof.

By $\{\varphi\}$ we denote the Riesz basis biorthogonal to the basis $\{\psi\}$ in Theorem 2.4.

Remark 2.5. The elements of the basis $\{\varphi\}$ which correspond to the part $\left\{\widehat{\psi}_{m}^{j, s}\right\}$ are the generalized eigenvectors $\left\{\widehat{\varphi}_{m}^{j, s}\right\}$ of the operator $\mathcal{A}$ (see (2.7)). So,

$$
\{\varphi\}=\left\{\varphi_{m, k}^{j, s},|k|>N\right\} \cup\left\{\widehat{\varphi}_{m}^{j, s}\right\} .
$$

Moreover, it is easy to show that there exists $N_{1}$ such that for any given $N>N_{1}$ and $m=1, \ldots, \ell$ the collection $\left\{\varphi_{m, k}^{j, s}, j=1, \ldots, \nu_{m} ; s=1, \ldots, p_{m, j}\right\}$ is a basis of $V_{m}^{(k)}$. The chosen basis $\{\varphi\}$ will be used in our further analysis of the steering conditions by the moment problem method. In this context, we notice that the construction of a proper basis becomes rather complicated only in the case when the spectrum of the matrix $A_{-1}$ is not simple and, as a consequence, the operator $\mathcal{A}$ may not possess a spectral Riesz basis. If all eigenvalues of $A_{-1}$ are simple, the basis $\{\varphi\}$ constructed in this section coincides with a spectral basis of $\mathcal{A}$.

3. Expansion of the steering condition in the Riesz basis. In order to use the results of section 2 , we assume that the matrix $A_{-1}$ is not singular.

Let us expand the steering condition (1.4) with respect to the basis $\{\varphi\}$ and to the biorthogonal basis $\{\psi\}$. Consider a state $x=\left(\begin{array}{c}y \\ z(\cdot)\end{array}\right) \in M_{2}$; this state is reachable at time $T$ if and only if

$$
\sum_{\varphi \in\{\varphi\}}\langle x, \psi\rangle \varphi=\sum_{\varphi \in\{\varphi\}} \int_{0}^{T}\left\langle\mathrm{e}^{\mathcal{A} t} \mathcal{B} u(t), \psi\right\rangle \mathrm{d} t \varphi, \quad u(\cdot) \in L_{2}\left(-1,0 ; \mathbb{C}^{r}\right) .
$$

Then the steering condition (1.4) can be substituted by the following system of equalities:

$$
\langle x, \psi\rangle=\int_{0}^{T}\left\langle\mathrm{e}^{\mathcal{A} t} \mathcal{B} u(t), \psi\right\rangle \mathrm{d} t, \quad \psi \in\{\psi\}, u(\cdot) \in L_{2}\left(-1,0 ; \mathbb{C}^{r}\right) .
$$

Let $\left\{b_{1}, \ldots, b_{r}\right\}$ be an arbitrary basis in $\operatorname{Im} B$, the image of the matrix $B$ and $\mathbf{b}_{i}=$ $\left(\begin{array}{c}b_{i} \\ 0\end{array}\right) \in M_{2}, i=1, \ldots, r$ (more precision on the choice of this basis will be given in section 6$)$. Then the right-hand side of (3.1) takes the form

$$
\int_{0}^{T}\left\langle\mathrm{e}^{\mathcal{A} t} \mathcal{B} u(t), \psi\right\rangle \mathrm{d} t=\sum_{i=1}^{r} \int_{0}^{T}\left\langle\mathrm{e}^{\mathcal{A} t} \mathbf{b}_{i}, \psi\right\rangle u_{i}(t) \mathrm{d} t .
$$

Let us omit the index $i$ for $\mathbf{b}_{i}$ and for any $\mathbf{b} \in\left\{\mathbf{b}_{1}, \ldots, \mathbf{b}_{r}\right\}$ transform the term $\left\langle\mathrm{e}^{\mathcal{A} t} \mathbf{b} u(t), \psi\right\rangle, \psi \in\left\{\psi_{m, k}^{j, s},|k|>N\right\}$ as follows:

$$
\begin{aligned}
\left\langle\mathrm{e}^{\mathcal{A} t} \mathbf{b}, \psi_{m, k}^{j, s}\right\rangle & =\left\langle\mathrm{e}^{\mathcal{A} t} \mathbf{b}, P_{m}^{(k) *} \widetilde{\psi}_{m, k}^{j, s}\right\rangle \\
& =\left\langle P_{m}^{(k)} \mathrm{e}^{\mathcal{A} t} \mathbf{b}, \widetilde{\psi}_{m, k}^{j, s}\right\rangle \\
& =\left\langle\widetilde{P}_{m}^{(k)} \mathrm{e}^{\widetilde{\mathcal{A}} t} \mathbf{b}, \widetilde{\psi}_{m, k}^{j, s}\right\rangle+\left\langle\left(P_{m}^{(k)} \mathrm{e}^{\mathcal{A} t}-\widetilde{P}_{m}^{(k)} \mathrm{e}^{\widetilde{\mathcal{A}} t}\right) \mathbf{b}, \widetilde{\psi}_{m, k}^{j, s}\right\rangle .
\end{aligned}
$$

Lemma 3.1. There exists a sequence $\left\{\alpha_{k}\right\}, \sum_{|k|>N} \alpha_{k}^{2}<\infty$, such that for all $m=1, \ldots, \ell ; j=1, \ldots, \nu_{m}$, and $s=1, \ldots, p_{m, j}$ the following estimates hold:

$$
\left|\left\langle\left(P_{m}^{(k)} \mathrm{e}^{\mathcal{A} t}-\widetilde{P}_{m}^{(k)} \mathrm{e}^{\tilde{\mathcal{A}} t}\right) \mathbf{b}, \widetilde{\psi}_{m, k}^{j, s}\right\rangle\right| \leq \frac{\alpha_{k}}{|k|}, \quad|k|>N, \quad t \in[0, T]
$$

Copyright (C) by SIAM. Unauthorized reproduction of this article is prohibited. 
Proof. Let us denote by $R(\lambda, \mathcal{A})$ and $R(\lambda, \widetilde{\mathcal{A}})$ the resolvents of the operators $\mathcal{A}$ and $\widetilde{\mathcal{A}}$. Taking into account the relation $(2.3)$ we can write

$$
\begin{aligned}
& \left|\left\langle\left(P_{m}^{(k)} \mathrm{e}^{\mathcal{A} t}-\widetilde{P}_{m}^{(k)} \mathrm{e}^{\widetilde{\mathcal{A}} t}\right) \mathbf{b}, \widetilde{\psi}_{m, k}^{j, s}\right\rangle\right| \\
& \quad=\left|\left\langle\frac{1}{2 \pi \mathrm{i}} \int_{L_{m}^{k}} \mathrm{e}^{\lambda t}(R(\lambda, \mathcal{A})-R(\lambda, \widetilde{\mathcal{A}})) \mathrm{d} \lambda \cdot \mathbf{b}, \widetilde{\psi}_{m, k}^{j, s}\right\rangle\right| \\
& \quad=\frac{1}{2 \pi}\left|\int_{L_{m}^{k}} \mathrm{e}^{\lambda t}\left\langle(R(\lambda, \mathcal{A})-R(\lambda, \widetilde{\mathcal{A}})) \mathbf{b}, \widetilde{\psi}_{m, k}^{j, s}\right\rangle \mathrm{d} \lambda\right| \\
& \quad \leq \frac{1}{2 \pi} \int_{L_{m}^{k}}\left|\mathrm{e}^{\lambda t}\right|\|(R(\lambda, \mathcal{A})-R(\lambda, \widetilde{\mathcal{A}})) \mathbf{b}\||| \widetilde{\psi}_{m, k}^{j, s} \||\mathrm{d} \lambda| \\
& \quad \leq C \int_{L_{m}^{k}}\left|\mathrm{e}^{\lambda t}\right|\|(R(\lambda, \mathcal{A})-R(\lambda, \widetilde{\mathcal{A}})) \mathbf{b}\||\mathrm{d} \lambda|
\end{aligned}
$$

with $C>0$. Now we need to estimate $\|(R(\lambda, \mathcal{A})-R(\lambda, \widetilde{\mathcal{A}})) \mathbf{b}\|$. In order to do that, we need to use an explicit expression for the resolvents of the operators $\mathcal{A}$ and $\widetilde{\mathcal{A}}$ given in [10, Proposition 2.2] (for the proof see [11] and also [5]). We obtain

$$
(R(\lambda, \mathcal{A})-R(\lambda, \widetilde{\mathcal{A}})) \mathbf{b}=\left(\begin{array}{c}
\left(I-A_{-1} \mathrm{e}^{-\lambda}\right)\left(\Delta_{\mathcal{A}}^{-1}(\lambda)-\Delta_{\widetilde{\mathcal{A}}}^{-1}(\lambda)\right) b \\
\mathrm{e}^{\lambda \theta}\left(\Delta_{\mathcal{A}}^{-1}(\lambda)-\Delta_{\widetilde{\mathcal{A}}}^{-1}(\lambda)\right) b
\end{array}\right)
$$

where

$$
\Delta_{\mathcal{A}}(\lambda)=\lambda I-\lambda \mathrm{e}^{-\lambda} A_{-1}+\lambda \int_{-1}^{0} \mathrm{e}^{\lambda s} A_{2}(s) \mathrm{d} s-\int_{-1}^{0} \mathrm{e}^{\lambda s} A_{3}(s) \mathrm{d} s,
$$

and, from that, $\Delta_{\widetilde{\mathcal{A}}}(\lambda)=\lambda I-\lambda \mathrm{e}^{-\lambda} A_{-1}$. From the relation

$$
\Delta_{\widetilde{\mathcal{A}}}^{-1}(\lambda)-\Delta_{\mathcal{A}}^{-1}(\lambda)=\Delta_{\mathcal{A}}^{-1}(\lambda)\left(\lambda \int_{-1}^{0} \mathrm{e}^{\lambda s} A_{2}(s) \mathrm{d} s+\int_{-1}^{0} \mathrm{e}^{\lambda s} A_{3}(s) \mathrm{d} s\right) \Delta_{\widetilde{\mathcal{A}}}^{-1}(\lambda),
$$

and using the estimates [11, formulas (25), (26)],

$$
\left\|\Delta_{\mathcal{A} \text { or } \widetilde{\mathcal{A}}}^{-1}(\lambda)\right\| \leq C_{1}|\lambda|^{-1}, \quad C_{1}>0, \quad \lambda \in L_{m}^{(k)}, \quad|k|>N, m=1, \ldots, \ell,
$$

we get the inequality

$$
\|(R(\lambda, \mathcal{A})-R(\widetilde{\lambda, \mathcal{A}})) \mathbf{b}\| \leq \frac{C_{2}}{|\lambda|^{2}}\left(|\lambda|\left\|\int_{-1}^{0} \mathrm{e}^{\lambda s} A_{2}(s) \mathrm{d} s\right\|+\left\|\int_{-1}^{0} \mathrm{e}^{\lambda s} A_{3}(s) \mathrm{d} s\right\|\right) .
$$

For $\lambda \in L_{m}^{(k)}$ we can put $\lambda=\widetilde{\lambda}+2 k \pi \mathrm{i}$, where $\tilde{\lambda} \in L_{m}^{(0)}=\left\{\xi:|\xi-\ln | \mu_{m}\left|+\mathrm{i} \arg \mu_{m}\right|=r\right\}$. This yields

$$
\begin{aligned}
\left\|\int_{-1}^{0} \mathrm{e}^{\lambda s} A_{2}(s) \mathrm{d} s\right\| & =\left\|\int_{-1}^{0} \mathrm{e}^{\tilde{\lambda} s} A_{2}(s) \mathrm{e}^{2 \pi \mathrm{i} k s} \mathrm{~d} s\right\|=C_{2}^{(k)}(\tilde{\lambda}), \\
\left\|\int_{-1}^{0} \mathrm{e}^{\lambda s} A_{3}(s) \mathrm{d} s\right\| & =\left\|\int_{-1}^{0} \mathrm{e}^{\tilde{\lambda} s} A_{3}(s) \mathrm{e}^{2 \pi \mathrm{i} k s} \mathrm{~d} s\right\|=C_{3}^{(k)}(\tilde{\lambda}),
\end{aligned}
$$

Copyright (c) by SIAM. Unauthorized reproduction of this article is prohibited. 
where

$$
\sum_{|k|>N}\left[C_{j}^{(k)}(\widetilde{\lambda})\right]^{2} \leq \int_{-1}^{0}\left|\mathrm{e}^{\widetilde{\lambda} s}\right|^{2}\left\|A_{j}(s)\right\|^{2} \mathrm{~d} s, \quad j=2,3 .
$$

Since for all values of the parameter

$$
\tilde{\lambda} \in \bigcup_{m=1}^{\ell} L_{m}^{(0)}
$$

the $L_{2}$-norm of the matrix functions $\mathrm{e}^{\widetilde{\lambda} s} A_{j}(s), j=2,3$, on the interval $[-1,0]$ are uniformly bounded, then there exists $\delta>0$ such that

$$
\sum_{|k|>N}\left(C_{j}^{(k)}(\widetilde{\lambda})\right)^{2} \leq \delta<\infty, \quad j=2,3, \quad \tilde{\lambda} \in \bigcup_{m=1}^{\ell} L_{m}^{(0)} .
$$

Finally, from (3.6) and (3.7), for $\tilde{\lambda} \in L_{m}^{(k)}$ we obtain

$$
\|(R(\lambda, \mathcal{A})-R(\lambda, \widetilde{\mathcal{A}})) \mathbf{b}\| \leq \frac{C_{2}}{|\widetilde{\lambda}+2 k \pi \mathrm{i}|}\left(|\widetilde{\lambda}+2 k \pi \mathrm{i}| C_{2}^{(k)}(\widetilde{\lambda})+C_{3}^{(k)}(\widetilde{\lambda})\right), \quad \tilde{\lambda} \in L_{m}^{(0)} .
$$

Then the inequalities (3.5), (3.6), and (3.9) give the validity of (3.4). The proof is complete.

Let us consider the first term on the right-hand side of (3.3). Since $\widetilde{\psi}_{m, k}^{j, s} \in W_{m}^{(k)}$ and due to Proposition 2.1, we have

$$
\begin{aligned}
\left\langle\widetilde{P}_{m}^{(k)} \mathrm{e}^{\widetilde{\mathcal{A}} t} \mathbf{b}, \widetilde{\psi}_{m, k}^{j, s}\right\rangle & =\left\langle\mathbf{b}, \mathrm{e}^{\widetilde{\mathcal{A}}^{*} t} \widetilde{P}_{m}^{(k) *} \widetilde{\psi}_{m, k}^{j, s}\right\rangle \\
& =\left\langle\mathbf{b}, \mathrm{e}^{\widetilde{\mathcal{A}}^{*} t} \widetilde{\psi}_{m, k}^{j, s}\right\rangle \\
& =\left(\left\langle\mathbf{b}, \widetilde{\psi}_{m, k}^{j, p_{m, j}}\right\rangle \frac{t^{p_{m, j}-s}}{\left(p_{m, j}-s\right) !}+\cdots+\left\langle\mathbf{b}, \widetilde{\psi}_{m, k}^{j, s}\right\rangle\right) \mathrm{e}^{\lambda_{m}^{(k)} t}
\end{aligned}
$$

Lemma 3.2. There exists a constant $\delta_{1}$ such that

$$
\left|\left\langle\mathbf{b}, \widetilde{\psi}_{m, k}^{j, s}\right\rangle\right| \leq \frac{\delta_{1}}{|k|}
$$

for all $|k|>N, m=1, \ldots, \ell, j=1, \ldots, \nu_{m}, s=1, \ldots, p_{m . j}$. Moreover, we have

$$
\left\langle\mathbf{b}, \widetilde{\psi}_{m, k}^{j, p_{m . j}}\right\rangle=\frac{1}{\bar{\lambda}_{m}^{(k)}}\left\langle b, C_{m, j}^{1}\right\rangle
$$

where $C_{m, j}^{1}$ are the eigenvectors of $A_{-1}$ corresponding to $\bar{\mu}_{m}$ and $\widetilde{\psi}_{m, k}^{j, p_{m . j}}$ are as in the formula (2.5) in Proposition 2.2 with $\beta_{m, k}^{j}=1$.

Proof. Taking into account (2.3), we get

$$
\begin{aligned}
\left|\left\langle\mathbf{b}, \widetilde{\psi}_{m, k}^{j, s}\right\rangle\right| & =\left|\left\langle\mathbf{b}, \widetilde{P}_{m}^{(k) *} \widetilde{\psi}_{m, k}^{j, s}\right\rangle\right| \\
& =\left|\left\langle\widetilde{P}_{m}^{(k)} \mathbf{b}, \widetilde{\psi}_{m, k}^{j, s}\right\rangle\right| \\
& =\frac{1}{2 \pi}\left|\int_{L_{m}^{(k)}}\left\langle R(\lambda, \widetilde{\mathcal{A}}) \mathbf{b}, \widetilde{\psi}_{m, k}^{j, s}\right\rangle \mathrm{d} \lambda\right| \\
& \leq C \int_{L_{m}^{(k)}}\|R(\lambda, \widetilde{\mathcal{A}}) \mathbf{b}\| \mathrm{d} \lambda, \quad C>0,
\end{aligned}
$$

Copyright (c) by SIAM. Unauthorized reproduction of this article is prohibited. 
where $C$ is a constant. The explicit expression of the resolvent $R(\lambda, \widetilde{\mathcal{A}})$ is given by $($ see $[10,11])$

$$
R(\lambda, \widetilde{\mathcal{A}}) \mathbf{b}=\left(\begin{array}{c}
\left(I-A_{-1} \mathrm{e}^{-\lambda}\right) \Delta_{\widetilde{\mathcal{A}}}^{-1}(\lambda) b \\
\mathrm{e}^{\lambda \theta} \Delta_{\tilde{\mathcal{A}}}^{-1}(\lambda) b
\end{array}\right)
$$

Since $\left\|\Delta_{\widetilde{\mathcal{A}}}^{-1}\right\| \leq C_{1}|\lambda|^{-1}$ with $C_{1}>0$ and for $\lambda \in L_{m}^{(k)},|k|>N, m=1, \ldots, \ell$, we obtain the estimate

$$
\|R(\lambda, \widetilde{\mathcal{A}}) \mathbf{b}\| \leq \frac{C_{2}}{|k|}, \quad C_{2}>0, \quad \lambda \in L_{m}^{(k)} .
$$

This, together with (3.12), leads to (3.11).

The second statement follows directly:

$$
\left\langle\mathbf{b}, \widetilde{\psi}_{m, k}^{j, p_{m . j}}\right\rangle=\left\langle\left(\begin{array}{c}
b \\
0
\end{array}\right), \widetilde{\psi}_{m, k}^{j, p_{m . j}}\right\rangle=\frac{1}{\bar{\lambda}_{m}^{(k)}}\left\langle b, C_{m, j}^{1}\right\rangle .
$$

This completes the proof.

Lemma 3.3. Assume that $\left\langle b, C_{m, j}^{s}\right\rangle=0, s=1, \ldots, r, r<p_{m, j}$; then

$$
\left\langle\mathbf{b}, \widetilde{\psi}_{m, k}^{j, p_{m, j}-r}\right\rangle=\frac{q_{m, j}^{r}}{\bar{\lambda}_{m}^{(k)}}\left\langle b, C_{m, j}^{r+1}\right\rangle
$$

where the coefficients $q_{m, j}^{r}$ do not depend on $k$.

Proof. This is a direct consequence of the relation (2.4).

Let us denote by $q_{m, k}^{j, s, d}(t)$ the polynomials

$$
q_{m, k}^{j, s, d}(t)=k\left(\frac{\left\langle\mathbf{b}_{d}, \widetilde{\psi}_{m, k}^{j, p_{m, j}}\right\rangle}{\left(p_{m, j}-s\right) !} t^{p_{m, j}-s}+\frac{\left\langle\mathbf{b}_{d}, \widetilde{\psi}_{m, k}^{j, p_{m, j}-1}\right\rangle}{\left(p_{m, j}-s-1\right) !} t^{p_{m, j}-s-1}+\cdots+\left\langle\mathbf{b}_{d}, \widetilde{\psi}_{m, k}^{j, s}\right\rangle\right)
$$

and by $f_{m, k}^{j, s, d}(t)$ the functions

$$
f_{m, k}^{j, s, d}(t)=k\left\langle\left(P_{m}^{(k)} \mathrm{e}^{\mathcal{A} t}-\widetilde{P}_{m}^{(k)} \mathrm{e}^{\widetilde{\mathcal{A}} t}\right) \mathbf{b}_{d}, \widetilde{\psi}_{m, k}^{j, s}\right\rangle .
$$

With these notations (and also due to (3.2), (3.3), and (3.10)), the infinite part of the system (3.1) corresponding to $\psi \in\left\{\psi_{m, k}^{j, s}\right\},|k|>N$, reads as

$$
k\left\langle\left(\begin{array}{c}
y_{T} \\
z_{T}(\cdot)
\end{array}\right), \psi_{m, k}^{j, s}\right\rangle=\sum_{d=1}^{r} \int_{0}^{T}\left(\mathrm{e}^{\lambda_{m}^{(k)} t} q_{m, k}^{j, s, d}(t)+f_{m, k}^{j, s, d}(t)\right) u_{d}(t) \mathrm{d} t .
$$

Moreover, due to Lemmas 3.1, 3.2, and 3.3, the functions (3.14) and (3.15) verify the following properties:

(P1) the coefficients of the polynomials $\{q(t)\}$ are uniformly bounded as $|k|>N$;

(P2) the set of leading coefficients of the nontrivial polynomials $\{q(t)\}$ does not have a limit point at 0 ;

(P3) $\sum_{|k|>N}\left|f_{m, k}^{j, s, d}(t)\right|^{2}<\alpha<\infty, t \in[0, T], \alpha>0$. 
Next we observe that if $\psi=\widehat{\psi}_{m}^{j, s}, m=1, \ldots, \ell_{N}, j=1, \ldots, \widehat{\mu}_{m}, s=1, \ldots, \widehat{p}_{m, j}$, then

$$
\begin{aligned}
\left\langle\mathrm{e}^{\mathcal{A} t} \mathbf{b}_{d}, \psi\right\rangle & =\left\langle\mathbf{b}_{d}, \mathrm{e}^{\mathcal{A}^{*} t} \psi\right\rangle \\
& =\widehat{q}_{m}^{j, s, d}(t) \mathrm{e}^{\widehat{\lambda}_{m} t},
\end{aligned}
$$

where

$$
\widehat{q}_{m}^{j, s, d}(t)=\left(\frac{\left\langle\mathbf{b}_{d}, \widehat{\psi}_{m, k}^{j, p_{m, j}}\right\rangle}{\left(\widehat{p}_{m, j}-s\right) !} t^{\widehat{p}_{m, j}-s}+\frac{\left\langle\mathbf{b}_{d}, \widehat{\psi}_{m, k}^{j, \widehat{p}_{m, j}-1}\right\rangle}{\left(\widehat{p}_{m, j}-s-1\right) !} t^{\widehat{p}_{m, j}-s-1}+\cdots+\left\langle\mathbf{b}_{d}, \widehat{\psi}_{m, k}^{j, s}\right\rangle\right) .
$$

Therefore, the finite part of the system (3.1) corresponding to $\psi \in\left\{\widehat{\psi}_{m}^{j, s}\right\}$ reads as

$$
\left\langle\left(\begin{array}{c}
y_{T} \\
z_{T}(\cdot)
\end{array}\right), \widehat{\psi}_{m}^{j, s}\right\rangle=\sum_{d=1}^{r} \int_{0}^{T} \mathrm{e}^{\widehat{\lambda}_{m} t} \widehat{q}_{m, k}^{j, s, d}(t) u_{d}(t) \mathrm{d} t .
$$

Thus, we observe that the state $\left(\begin{array}{c}y_{T} \\ z_{T}(\cdot)\end{array}\right) \in M_{2}$ is reachable from 0 at the time $T>0$ if and only if the equalities (3.16) and (3.17) hold for some controls $u_{d}(\cdot) \in L_{2}(0, T), d=$ $1, \ldots, r$. These equalities pose a kind of moment problem, which is the main object of our further analysis.

4. The problem of moments and the Riesz basis property. In this section, we recall the general properties of the problem of moments that will be applied to the analysis of the problem (3.16)-(3.17) given in section 3.

Consider a collection of functions $\left\{g_{k}(t), t \in\left[0, \infty[\}_{k \in \mathbb{N}}\right.\right.$ assuming that for any $k \in \mathbb{N}, T>0, g_{k}(\cdot) \in L_{2}(0, T)$, and consider the following problem of moments:

$$
s_{k}=\int_{0}^{T} g_{k}(t) u(t) \mathrm{d} t, \quad k \in \mathbb{N} .
$$

We start with the following well-known fact, which is a consequence of Bari theorem [4, Chapter 6] and [17, Chapter 4] (see also [14] for a direct proof and the references therein).

Proposition 4.1. The following statements are equivalent:

(i) For the scalars $s_{k}, k \in \mathbb{N}$, the problem (4.1) has a solution $u(\cdot) \in L_{2}(0, T)$ if and only if $\left\{s_{k}\right\} \in \ell_{2}$, i.e., $\sum_{k \in \mathbb{N}} s_{k}^{2}<\infty$;

(ii) the family $\left\{g_{k}(t)\right\}_{k \in \mathbb{N}}, t \in[0, T]$, forms a Riesz basis in the closure of its linear span

$$
\mathrm{Cl} \operatorname{Lin}\left\{g_{k}(t), k \in \mathbb{N}\right\} .
$$

Using this proposition, we can prove the following result.

Proposition 4.2. Let us denote by $\mathcal{L}(0, T)$ the closed subspace

$$
\mathrm{Cl} \operatorname{Lin}\left\{g_{k}(t), k \in \mathbb{N}\right\} \subset L_{2}\left(T_{0}, T\right) .
$$

Let us suppose that for some $T_{1}>0$ the functions $\left\{g_{k}(t)\right\}_{k \in \mathbb{N}}, t \in\left[0, T_{1}\right]$, form a Riesz basis in $\mathcal{L}\left(0, T_{1}\right) \subset L_{2}\left(0, T_{1}\right)$ and $\operatorname{codim} \mathcal{L}\left(0, T_{1}\right)<\infty$. Then for any $0<T<T_{1}$, there exists an infinite-dimensional subspace $\ell^{T} \subset \ell_{2}$ such that the problem of moments (4.1) is unsolvable for $\left\{s_{k}\right\} \in \ell^{T}$ if $\left\{s_{k}\right\} \neq\{0\}$.

Copyright (c) by SIAM. Unauthorized reproduction of this article is prohibited. 
Proof. We introduce for all $T>0$ the operator $Q_{T}: L_{2}(0, T) \rightarrow \ell_{2}$ given by

$$
Q_{T} u(\cdot)=\left\{\int_{0}^{T} g_{k}(t) u(t) \mathrm{d} t\right\}_{k \in \mathbb{N}} .
$$

This gives $Q_{T_{1}}\left(L_{2}\left(0, T_{1}\right)\right)=\ell_{2}$ by Proposition 4.1. The operator $Q_{T_{1}}$ is bounded due to the closed graph theorem. It is easy to see that the adjoint operator $Q_{T_{1}}^{*}$ acts as

$$
Q_{T_{1}}^{*}\left\{s_{k}\right\}_{k \in \mathbb{N}}=\sum_{k \in \mathbb{N}} s_{k} g_{k}(t) \in \mathcal{L}\left(0, T_{1}\right)
$$

Let us denote now by $Q_{1}: \mathcal{L}\left(0, T_{1}\right) \rightarrow \ell_{2}$ the one-to-one operator defined as follows:

$$
Q_{1} u(\cdot)=\left\{c_{k}\right\} \quad \text { for } \quad u(\cdot)=\sum_{k \in \mathbb{N}} c_{k} g_{k}(\cdot),\left\{c_{k}\right\}, \in \ell_{2} .
$$

Now consider the decomposition $L_{2}\left(0, T_{1}\right)=X_{1} \oplus X_{2}$, where

$$
X_{1}=\{u(\cdot): u(t) \equiv 0, t \in[0, T]\}, \quad X_{2}=\left\{u(\cdot): u(t) \equiv 0, t \in\left[T, T_{1}\right]\right\},
$$

and observe that since $\operatorname{codim} \mathcal{L}\left(0, T_{1}\right)<\infty$, then the intersection $X=X_{1} \cup \mathcal{L}\left(0, T_{1}\right)$ is an infinite-dimensional subspace in $L_{2}\left(0, T_{1}\right)$. Finally, let us denote $\ell^{T}=Q_{1}(X)$. The above considerations prove that this subspace is infinite dimensional. Taking $u(\cdot) \in X_{2}$ and $\left\{s_{k}\right\} \in \ell^{T}$, we obtain

$$
\left\langle Q_{T_{1}} u(\cdot),\left\{s_{k}\right\}\right\rangle=\left\langle u(\cdot), Q_{T}^{*}\left\{s_{k}\right\}\right\rangle=\int_{0}^{T} u(t) \sum_{k \in \mathbb{N}} s_{k} g_{k}(t) \mathrm{d} t=0
$$

because $\sum_{k \in \mathbb{N}} s_{k} g_{k}(t) \in X_{1}$. Thus $Q_{T_{1}}\left(X_{2}\right) \perp \ell^{T}$ and, therefore, (4.1) is unsolvable for $\left\{s_{k}\right\} \in \ell^{T}$ if $\left\{s_{k}\right\} \neq\{0\}$.

Proposition 4.3. Let us consider the moment problem

$$
s_{k}=\sum_{d=1}^{r} \int_{0}^{T} g_{k}^{d}(t) u_{d}(t) \mathrm{d} t, \quad k \in \mathbb{N}
$$

with the assumption

$$
\sum_{k \in \mathbb{N}} \int_{0}^{T}\left|g_{k}^{d}(t)\right|^{2} \mathrm{~d} t<\infty, \quad d=1, \ldots, r .
$$

Then the set $S_{0, T}$ of sequences $\left\{s_{k}\right\}$ for which problem (4.3) is solvable is a nontrivial submanifold of $\ell_{2}$, i.e., $S_{0, T} \neq \ell_{2}$.

Proof. Let us introduce the operator $Q_{T}^{r}: L_{2}^{r}(0, T) \rightarrow \ell^{2}$ defined by

$$
Q_{T}^{r} u(\cdot)=\left\{s_{k}\right\}_{k \in \mathbb{N}}=\left\{\sum_{d=1}^{r} \int_{0}^{T} g_{k}^{d}(t) u_{d}(t) \mathrm{d} t\right\}_{k \in \mathbb{N}}, \quad u(\cdot)=\left(u_{1}(\cdot), \ldots, u_{r}(\cdot)\right) .
$$


Then, if $\|u(\cdot)\| \leq 1$, we obtain

$$
\begin{aligned}
\sum_{k=N}^{\infty}\left|s_{k}\right|^{2} & =\sum_{d=1}^{r} \sum_{k=N}^{\infty}\left|\int_{0}^{T} g_{k}^{d}(t) u_{d}(t)\right|^{2} \mathrm{~d} t \\
& \leq \sum_{d=1}^{r} \sum_{k=N}^{\infty} \int_{0}^{T}\left|g_{k}^{d}(t)\right|^{2} \mathrm{~d} t
\end{aligned}
$$

and then $\sum_{k=N}^{\infty}\left|s_{k}\right|^{2} \rightarrow 0$ as $N \rightarrow \infty$. This means that the set $\left\{Q_{T}^{r} u(\cdot), \| u(\cdot) \mid \leq 1\right\}$ satisfies the criterion of compactness in $\ell^{2}$ (see, for example, [7, Chapter 5]). Hence $Q_{T}^{r}$ is a compact operator and therefore $\operatorname{Im} Q_{T}^{r} \neq \ell_{2}$.

In the following, our analysis will be based on the theory of families of exponential developed by Avdonin and Ivanov in [1]. We are particularly interested in the basis properties of such families.

Let $\delta_{1}, \ldots, \delta_{\ell}$ be different, modulus $2 \pi \mathrm{i}$, complex numbers, and let $m_{1}, \ldots, m_{\ell}$ and $N$ be natural integers. Let us denote by $\widetilde{\mathcal{E}}_{N}$ the family

$$
\widetilde{\mathcal{E}}_{N}=\left\{\mathrm{e}^{\left(\delta_{s}+2 \pi \mathrm{i} k\right) t}, t \mathrm{e}^{\left(\delta_{s}+2 \pi \mathrm{i} k\right) t}, \ldots, t^{m_{s}-1} \mathrm{e}^{\left(\delta_{s}+2 \pi \mathrm{i} k\right) t}\right\}_{\substack{|k|>N \\ s=1, \ldots, \ell}} .
$$

Next, let $\varepsilon_{1}, \ldots, \varepsilon_{r}$ be another collection of different complex numbers such that $\varepsilon_{j} \neq$ $\delta_{s}+2 \pi \mathrm{i} k, j=1, \ldots, r ; s=1, \ldots, \ell ;|k|>N$, and let $m_{1}^{\prime}, \ldots, m_{r}^{\prime}$ be positive integers. Let us denote by $\mathcal{E}_{0}$ the collection

$$
\mathcal{E}_{0}=\left\{\mathrm{e}^{\varepsilon_{j} t}, t \mathrm{e}^{\varepsilon_{j} t}, \ldots, t^{m_{s}^{\prime}-1} \mathrm{e}^{\varepsilon_{j} t}\right\}_{j=1, \ldots, r} .
$$

The following theorem is the main tool of our further analysis.

THEOREM 4.4. (i) If $\sum_{j=1}^{r} m_{j}^{\prime}=(2 N+1) \sum_{s=1}^{\ell} m_{s}$, then the family $\mathcal{E}=\widetilde{\mathcal{E}}_{N} \cup \mathcal{E}_{0}$ constitutes a Riesz basis in $L_{2}\left(0, \sum_{s=1}^{\ell} m_{s}\right)$.

(ii) If $T>\sum_{s=1}^{\ell} m_{s}$, then independently of the number of elements in $\mathcal{E}_{0}$, the family $\mathcal{E}$ forms a Riesz basis of the closure of its linear span in the space $L_{2}(0, T)$.

Proof. (i) We make use of [1, Theorem II.4.23]. According to this theorem, let us consider the complex function

$$
f(z)=\mathrm{e}^{\mathrm{i} z} \sum_{s=1}^{\ell} m_{s} \prod_{s=1}^{\ell}\left(\sin \left(\frac{z}{2}-\frac{\delta_{s}}{2}\right)\right)^{m_{s}} R(z),
$$

where

$$
R(z)=\prod_{j=1}^{r}\left(z-\varepsilon_{j}\right)^{m_{j}^{\prime}}\left(\prod_{\substack{s=1, \ldots, \ell \\|k| \leq N}}\left(z-\delta_{s}-2 \pi \mathrm{i} k\right)^{m_{s}}\right)^{-1} \rightarrow 1 \quad \text { as } \quad z \rightarrow \infty .
$$

One can easily verify that $f(z)$ extended to the points $\delta_{s}+2 \pi \mathrm{i} k, s=1, \ldots, \ell,|k| \leq N$, by continuity, is an entire function of the sine type (see [1, Definition II.1.27] and also [17, section 4.5]). Representing

$$
\prod_{s=1}^{\ell}\left(\sin \left(\frac{z}{2}-\frac{\delta_{s}}{2}\right)\right)^{m_{s}}=\prod_{s=1}^{\ell}\left(\frac{\mathrm{e}^{\frac{\mathrm{i} z}{2}} \mathrm{e}^{\frac{-\mathrm{i} \delta_{s}}{2}}-\mathrm{e}^{\frac{-\mathrm{i} z}{2} \mathrm{e}^{\frac{\mathrm{i} \delta_{s}}{2}}}}{2 \mathrm{i}}\right)^{m_{s}}
$$

Copyright (c) by SIAM. Unauthorized reproduction of this article is prohibited. 
we get

$$
\prod_{s=1}^{\ell}\left(\sin \left(\frac{z}{2}-\frac{\delta_{s}}{2}\right)\right)^{m_{s}}=C_{0} \mathrm{e}^{\frac{\mathrm{i} z}{2} \sum_{s=1}^{\ell} m_{s}}+C_{1} \mathrm{e}^{\frac{-\mathrm{i} z}{2} \sum_{s=1}^{\ell} m_{s}}+\sum_{j=2}^{N_{0}} C_{j} \mathrm{e}^{\mathrm{i} z q_{j}},
$$

where $C_{j}$ are constants and $q_{j} \in\left\{-\frac{1}{2} \sum_{s=1}^{\ell} m_{s}, \frac{1}{2} \sum_{s=1}^{\ell} m_{s}\right\}, j=2, \ldots, N_{0}$. And then the growth indicator of the function $f$ (see [1, Paragraph II.1.4.2]) is of the form

$$
\begin{aligned}
h_{f}(\phi) & =\lim _{\rho \rightarrow \infty} \sup \frac{1}{\rho} \ln \left|f\left(\rho \mathrm{e}^{\mathrm{i} \phi}\right)\right| \\
& =\lim _{\rho \rightarrow \infty} \sup \frac{1}{\rho} \ln \left|C_{0} \mathrm{e}^{\mathrm{i} z \sum_{s=1}^{\ell} m_{s}}+C_{1}+\sum_{j=2}^{N_{0}} C_{j} \mathrm{e}^{\mathrm{i} z\left(q_{j}+\frac{1}{2} \sum_{s=1}^{\ell} m_{s}\right)}\right| \\
& = \begin{cases}0, & \phi \in[0, \pi], \\
-\sum_{s=1}^{\ell} m_{s} \sin \phi, & \phi \in[-\pi, 0] .\end{cases}
\end{aligned}
$$

Therefore, the indicator diagram is $G_{f}=\left[-\mathrm{i} \sum_{s=1}^{\ell} m_{s}, 0\right]$. Finally, observe that the set of zeros of $f$ is exactly

$$
\left\{\delta_{s}+2 \pi \mathrm{i} k\right\}_{\substack{s=1, \ldots, \ell \\|k|>N}} \bigcup\left\{\varepsilon_{j}\right\}_{j=1, \ldots, r},
$$

the roots $\delta_{s}+2 \pi \mathrm{i} k$ are of multiplicity $m_{s}$, and the roots $\varepsilon_{j}$ are of multiplicity $m_{j}^{\prime}$. To summarize, we conclude that $f(z)$ is a generating function (see [1, Definition II.4.21]) of the family $\mathcal{E}$ on the interval $\left[0, \sum_{s=1}^{\ell} m_{s}\right]$ and, therefore, this family is a Riesz basis of $L_{2}\left(0, \sum_{s=1}^{\ell} m_{s}\right)$. The statement is proved. that

(ii) Let us denote $\gamma=T-\sum_{s=1}^{\ell} m_{s}>0$ and choose a complex number $\mu$ such

$$
\mu+\frac{2 \pi \mathrm{i} m}{\gamma} \neq \delta_{s}+2 \pi \mathrm{i} k \quad \text { and } \quad \mu+\frac{2 \pi \mathrm{i} m}{\gamma} \neq \varepsilon_{j}
$$

for all $m, k \in \mathbb{Z}, s=1, \ldots, \ell, j=1, \ldots, r$. Let us put $m^{\prime}=\sum_{j=1}^{r} m_{j}^{\prime}$ and

$$
\begin{aligned}
\mathcal{E}_{1}^{\left(m^{\prime}\right)} & =\left\{\mathrm{e}^{\left(\mu+\frac{2 \pi \mathrm{i} m}{\gamma}\right) t}\right\}_{m \in \mathbb{Z} \backslash\left\{1, \ldots, m^{\prime}\right\}}, \\
\widetilde{\mathcal{E}}_{N} & =\left\{\mathrm{e}^{\left(\delta_{s}+2 \pi \mathrm{i} k\right) t}, t \mathrm{e}^{\left(\delta_{s}+2 \pi \mathrm{i} k\right) t}, \ldots, t^{m_{s}-1} \mathrm{e}^{\left(\delta_{s}+2 \pi \mathrm{i} k\right) t}\right\}_{\substack{|k|>N \\
s=1, \ldots, \ell}}
\end{aligned}
$$

and consider the family

$$
\widetilde{\mathcal{E}}_{N} \cup \mathcal{E}_{0} \cup \mathcal{E}_{1}^{\left(m^{\prime}\right)} .
$$

Now let us introduce a complex function of the sine type given by

$$
f_{1}(z)=\mathrm{e}^{\mathrm{i}\left(\frac{z}{2} \sum_{s=1}^{\ell} m_{s}+\gamma\right)} \prod_{s=1}^{\ell}\left(\sin \left(\frac{z}{2}-\frac{\delta_{s}}{2}\right)\right)^{m_{s}} R_{1}(z) \sin \gamma\left(\frac{z}{2}-\frac{\mu}{2}\right),
$$

where

$$
R_{1}(z)=\prod_{j=1}^{r}\left(z-\varepsilon_{j}\right)^{m_{j}^{\prime}}\left(\prod_{m=1}^{m_{j}^{\prime}}\left(z-\mu-\frac{2 \pi \mathrm{i} m}{\gamma}\right)\right)^{-1} \rightarrow 1 \quad \text { as } \quad z \rightarrow \infty .
$$

Copyright $@$ ㅇ by SIAM. Unauthorized reproduction of this article is prohibited. 
Then, by arguments analogous to those given in the proof of part (i), it follows that $f_{1}(z)$ is a generating function of $\widetilde{\mathcal{E}}_{N} \cup \mathcal{E}_{0} \cup \mathcal{E}_{1}^{\left(m^{\prime}\right)}$ on the interval $[0, T]$. Therefore, this family forms a Riesz basis of $L_{2}(0, T)$. Now, since $\mathcal{E} \subset \widetilde{\mathcal{E}}_{N} \cup \mathcal{E}_{0} \cup \mathcal{E}_{1}^{\left(m^{\prime}\right)}$, this means that $\mathcal{E}$ forms a Riesz basis in $\mathrm{Cl} \operatorname{Lin} \mathcal{E} \subset L_{2}(0, T)$. The proof of the theorem is complete.

Now we apply Theorem 4.4 to the collection of functions appearing in (3.16). Let us fix $d \in\{1, \ldots, r\}$ and choose an arbitrary subset $L \subset\{1, \ldots, \ell\}$. Next, for any $m \in L$ we choose $j(m) \in\left\{1, \ldots, \nu_{m}\right\}$ and denote $J(L)=\{j(m)\}_{m \in L}$. Finally, for any couple $(m, j(m)), m \in L$, we put $\pi_{m, j(m)}=\operatorname{deg} q_{m, k}^{j(m), 1, d}(t)+1$. Let us recall that from (3.14) and Lemmas 3.2 and 3.3 it follows that this degree does not depend on $k$.

THEOREM 4.5. For any choice of $d, L, J(L)$, for any $p_{m, j(m)}^{\prime}$, such that $1 \leq$ $p_{m, j(m)}^{\prime} \leq \pi_{m, j(m)}$, and for any $T \geq n^{\prime}=\sum_{m \in L} p_{m, j(m)}^{\prime}$ the collection of functions

$$
\Phi_{1}=\left\{\mathrm{e}^{\lambda_{m}^{(k)} t} q_{m, k}^{j(m), s, d}(t),|k|>N ; m \in L ; s=\pi_{m, j(m)}-p_{m, j(m)}^{\prime}+1, \ldots, \pi_{m, j(m)}\right\}
$$

constitutes a Riesz basis of $\mathrm{Cl} \operatorname{Lin} \Phi_{1}$ in $L_{2}(0, T)$.

If in addition $N$ is large enough, then the family

$$
\Phi_{2}=\left\{\mathrm{e}^{\lambda_{m}^{(k)} t} q_{m, k}^{j(m), s, d}(t)+f_{m, k}^{j(m), s, d}(t)\right\}_{|k|>N ; m \in L ; s=\pi_{m, j(m)}-p_{m, j(m)}^{\prime}+1, \ldots, \pi_{m, j(m)}}
$$

also forms a Riesz basis of $\mathrm{Cl} \operatorname{Lin} \Phi_{2}$ in $L_{2}(0, T)$.

If $T=n^{\prime}$, the subspaces $\mathrm{Cl} \operatorname{Lin} \Phi_{1}$ and $\mathrm{Cl} \operatorname{Lin} \Phi_{2}$ are of finite codimension $(2 N+$ 1) $n^{\prime}$ in $L_{2}\left(0, n^{\prime}\right)$.

Proof. Consider the linear operator $\mathcal{T}: \operatorname{Lin} \Phi_{1} \rightarrow \operatorname{Lin} \Phi_{1}$ defined on the elements of $\Phi_{1}$ by the equalities

$$
\mathcal{T}\left(\mathrm{e}^{\lambda_{m}^{(k)} t} q_{m, k}^{j(m), s, d}(t)\right)=\mathrm{e}^{\lambda_{m}^{(k)}} t t^{p_{m, j(m)}^{\prime}-s}
$$

for $|k|>N ; m \in L ; s=\pi_{m, j(m)}-p_{m, j(m)}^{\prime}+1, \ldots, \pi_{m, j(m)}$. It follows from the properties (P1) and (P2) (see section 3) and Theorem 4.4 that the operator $\mathcal{T}$ is bounded in the sense of $L_{2}(0, T)$ and its extension to $L=\mathrm{Cl} \operatorname{Lin} \Phi_{1}$ is a bounded one-to-one operator from $L$ to $L$. Hence, since the images of the elements of $\Phi_{1}$ form a Riesz basis of $L$ (Theorem 4.4), then $\Phi_{1}$ is also a Riesz basis of this subspace of $L_{2}(0, T)$.

Next, let us introduce in $L_{2}(0, T)$ an equivalent norm $\|\cdot\|_{1}$ in which the system $\Phi_{1}$ becomes orthonormal. Let $\Phi_{1}^{c}$ be an orthonormal complement of the basis $\Phi_{1}$ to a basis of $L_{2}(0, T)$. Now using the property (P3), we choose the scalar $N$ large enough so that

$$
\sum_{\substack{|k|>N \\ \mid \in L \\ s \in I_{m}}}\left\|f_{m, k}^{j(m), s, d}\right\|_{1}^{2} \leq C \sum_{\substack{|k|>N \\ \mid<\in L \\ s \in I_{m}}}\left\|f_{m, k}^{j(m), s, d}\right\|_{L_{2}}^{2}<1,
$$

where $I_{m}=\pi_{m, j(m)}-p_{m, j(m)}^{\prime}+1, \ldots, \pi_{m, j(m)}$. Then $\Phi_{2} \cup \Phi_{1}^{c}$ is quadratically close in $\|\cdot\|_{1}$ to the orthonormal system $\Phi_{1} \cup \Phi_{1}^{c}$ with a quadratic distance less than 1 . This means that $\Phi_{2} \cup \Phi_{1}^{c}$ forms also a Riesz basis in $L_{2}(0, T)$ (see Gohberg and Krein [4]). As a consequence, $\Phi_{2}$ is a Riesz basis in $\operatorname{Cl} \operatorname{Lin} \Phi_{2}$.

Finally, let us observe that in the case $T=n^{\prime}$ the space $L$, which is also presented as

$L=\mathrm{Cl} \operatorname{Lin}\left\{\mathrm{e}^{\lambda_{m}^{(k)} t} t^{p_{m, j(m)}^{\prime}-s},|k|>N ; m \in L, s=\pi_{m, j(m)}-p_{m, j(m)}^{\prime}+1, \ldots, \pi_{m, j(m)}\right\}$, 
is of codimension $(2 N+1) n^{\prime}$ in $L_{2}(0, T)$ (see Theorem 4.4). Then $\Phi_{1}^{c}$ consists of exactly $(2 N+1) n^{\prime}$ elements. The proof is complete.

5. Analysis of the controllability for a single control. Let us study the solvability of the systems of equalities (3.16) and (3.17). We assume again that the matrix $A_{-1}$ is not singular, $\operatorname{det} A_{-1} \neq 0$.

Consider the sequence of functions

$$
\begin{aligned}
\left\{\int_{0}^{T} \mathrm{e}^{\lambda_{m}^{(k)} t} q_{m, k}^{j(m), s, d}(t)+f_{m, k}^{j(m), s, d}(t) \mathrm{d} t\right\} & \\
= & \left\{\int_{0}^{T} \mathrm{e}^{\lambda_{m}^{(k)} t} q_{m, k}^{j(m), s, d}(t) \mathrm{d} t+\int_{0}^{T} f_{m, k}^{j(m), s, d}(t) \mathrm{d} t\right\}
\end{aligned}
$$

for $|k|>N, s=1, \ldots, p_{m, j(m)}$, and any fixed $d, m, j$ and $u(\cdot) \in L_{2}(0, T)$. It follows from Theorem 4.5 that all nonzero functions of the collection

$$
\left\{\mathrm{e}^{\lambda_{m}^{(k)} t} q_{m, k}^{j(m), s, d}(t),|k|>N ; s=1, \ldots, p_{m, j}\right\}
$$

form a Riesz basis of their linear span in $L_{2}\left(0, T^{\prime}\right)$ if $T^{\prime}$ is large enough. Therefore, by Proposition 4.1, the first term of (5.1) belongs to the class $\ell_{2}$. On the other hand, the second term also belongs to $\ell_{2}$ due to Proposition 4.3. This gives the following proposition.

Proposition 5.1. If the state $\left(\begin{array}{c}y_{T} \\ z_{T}(\cdot)\end{array}\right)$ is reachable from 0 by the system (1.3), then it satisfies the following equivalent conditions:

(C1) $\sum_{\substack{|k|>N \\ m, j, s}} k^{2}\left|\left\langle\left(\begin{array}{c}y_{T}(\cdot) \\ z_{T}(\cdot)\end{array}\right), \psi_{m, k}^{j, s}\right\rangle\right|^{2}<\infty$,

(C2) $\sum_{\substack{|k|>N \\ m=1, \ldots, \ell}} k^{2}\left\|P_{m}^{(k)}\left(\begin{array}{c}y_{T} \\ z_{T}(\cdot)\end{array}\right)\right\|^{2}<\infty$

(C3) $\left(\begin{array}{c}y_{T} z_{T}(\cdot) \\ z_{T}\end{array}\right) \in \mathcal{D}(\mathcal{A})$.

Proof. The condition (C1) follows from the previous consideration. Note that actually the validity of $(\mathrm{C} 1)$ does not depend on the choice of the basis $\{\psi\}$. In fact, we can observe that

$$
P_{m}^{(k)}\left(\begin{array}{c}
y_{T} \\
z_{T}(\cdot)
\end{array}\right)=\sum_{\substack{j=1, \ldots, \nu_{m} \\
s=1, \ldots, p_{m, j}}}\left\langle\left(\begin{array}{c}
y_{T} \\
z_{T}(\cdot)
\end{array}\right), \psi_{m, k}^{j, s}\right\rangle \phi_{m, k}^{j, s}
$$

From here and since $\{\psi\}$ is a Riesz basis [14], we deduce that there exist two constants $c$ and $C$ (independently of $m$ and $k$ ) such that

$$
c^{2} \sum_{j, s}\left|\left\langle\left(\begin{array}{c}
y_{T} \\
z_{T}(\cdot)
\end{array}\right), \psi_{m, k}^{j, s}\right\rangle\right|^{2} \leq\left\|P_{m}^{(k)}\left(\begin{array}{c}
y_{T} \\
z_{T}(\cdot)
\end{array}\right)\right\|^{2} \leq C^{2}\left|\left\langle\left(\begin{array}{c}
y_{T} \\
z_{T}(\cdot)
\end{array}\right), \psi_{m, k}^{j, s}\right\rangle\right|^{2}
$$

and this gives the equivalence between $(\mathrm{C} 1)$ and $(\mathrm{C} 2)$. Let us show now that $(\mathrm{C} 1)$ and $(\mathrm{C} 2)$ are equivalent to $(\mathrm{C} 3)$.

First of all, we notice that from the explicit form of the resolvent $R(\lambda, \mathcal{A})$ given in [11, Proposition 1] and by arguments and estimates given in the proof of [11, Theorem 2.9], it follows that there exists a constant $C$ such that

$$
|| R(\lambda, \mathcal{A})\left|\leq C, \quad \lambda \in L_{m}^{(k)}, \quad\right| k \mid>N, m=1, \ldots, \ell .
$$

Copyright (c) by SIAM. Unauthorized reproduction of this article is prohibited. 
Let $\mathcal{A}_{m}^{(k)}: V_{m}^{(k)} \rightarrow V_{m}^{(k)}$ be the restriction of the operator $\mathcal{A}$ to its invariant subspace $V_{m}^{(k)}$. Then due to (5.3) we have

$$
\begin{aligned}
&\left\|\mathcal{A}_{m}^{(k)} v\right\| \leq \int_{L_{m}^{(k)}}|\lambda|\|R(\lambda, \mathcal{A})\|\|v\||\mathrm{d} \lambda| \leq C_{1}|k|\|v\|, \\
&\left\|\left(\mathcal{A}_{m}^{(k)}\right)^{-1} v\right\| \leq \int_{L_{m}^{(k)}} \frac{1}{|\lambda|}\|R(\lambda, \mathcal{A})\|\|v\|\left\|\mathrm{d} \lambda \mid \leq \frac{C_{1}^{\prime}}{|k|}\right\| v \|,
\end{aligned}
$$

where $v \in V_{m}^{(k)}$ and the constants $C_{1}, C_{1}^{\prime}$ do not depend on $m, k$. From this, one can obtain for $v \in V_{m}^{(k)}$ the inequality

$$
\frac{1}{C_{1}^{\prime}}\|v\| \leq \frac{1}{k}\left\|\mathcal{A}_{m}^{(k)} v\right\| \leq C_{1} \| v \mid
$$

With our notations, the condition (C3) is obviously equivalent to

$$
\sum_{\substack{|k|>N \\
m=1, \ldots, \ell}}\left\|\mathcal{A}_{m}^{(k)} P_{m}^{(k)}\left(\begin{array}{c}
y_{T} \\
z_{T}(\cdot)
\end{array}\right)\right\|^{2}<\infty .
$$

But, on the other hand, due to (5.4) this condition is equivalent to (C2). This completes the proof.

From Proposition 5.1 it follows once more, as was pointed out in the introduction (see also [5]), that the set $\mathcal{R}_{T}$ of the states reachable from 0 by virtue of the system (1.3) and controls from $L_{2}(0, T)$ is always a subset of $\mathcal{D}(\mathcal{A})$. This justifies also Definition 1.1 given in the introduction: the system (1.3) is said to be null-controllable at the time $T$ if $\mathcal{R}_{T}=\mathcal{D}(\mathcal{A})$. Next, we give the necessary conditions of null-controllability.

ThEOREM 5.2. Assume that the system (1.3) is null-controllable by controls from $L_{2}(0, T)$ for some $T>0$. Then the following two conditions hold.

(i) There is no $\lambda \in \mathbb{C}$ and $y \in \mathbb{C}^{n}, y \neq 0$, such that $\Delta_{\mathcal{A}}^{*}(\lambda) y=0$ and $B^{*} y=0$, where

$$
\Delta_{\mathcal{A}}^{*}(\lambda)=\lambda I-\lambda \mathrm{e}^{-\lambda} A_{-1}^{*}-\lambda \int_{-1}^{0} \mathrm{e}^{\lambda s} A_{2}^{*}(s) \mathrm{d} s-\int_{-1}^{0} \mathrm{e}^{\lambda s} A_{3}^{*}(s) \mathrm{d} s,
$$

or equivalently $\operatorname{rank}\left(\Delta_{\mathcal{A}}(\lambda) \quad B\right)=n$ for all $\lambda \in \mathbb{C}$.

(ii) There is no $\mu \in \sigma\left(A_{-1}\right)$ and $y \in \mathbb{C}^{n}, y \neq 0$, such that $A_{-1}^{*} y=\bar{\mu} y$ and $B^{*} y=0$, or equivalently $\operatorname{rank}\left(\begin{array}{llll}B & A_{-1} B & \cdots & A_{-1}^{n-1} B\end{array}\right)=n$.

First we prove the following lemma.

Lemma 5.3. Condition (i) of Theorem 5.2 is equivalent to the following condition:

$\left(\mathrm{i}^{\prime}\right)$ There is no eigenvector $g$ of the adjoint operator $\mathcal{A}^{*}$ belonging to Ker $\mathcal{B}^{*}$.

Proof of Lemma 5.3. We make use of the following explicit form of $\mathcal{A}^{*}$ :

$$
\mathcal{A}^{*}\left(\begin{array}{c}
y \\
z(\cdot)
\end{array}\right)=\left(\begin{array}{c}
A_{2}^{*}(0) y+z(0) \\
-\frac{\mathrm{d}}{\mathrm{d} \theta}\left(z(\theta)+A_{2}^{*}(\theta) y\right),+A_{3}^{*}(\theta) y
\end{array}\right)
$$

with the domain

$$
\begin{array}{r}
\mathcal{D}\left(\mathcal{A}^{*}\right)=\left\{(y, z(\cdot)) \in M_{2}: z(\theta)+A_{2}^{*}(\theta) y \in H^{1}\left([-1,0], \mathbb{C}^{n}\right),\right. \\
\left.\left(A_{-1}^{*} A_{2}^{*}(0)-A_{2}^{*}(-1)\right) y=z(-1)-A_{-1}^{*} z(0)\right\} .
\end{array}
$$

Copyright $\odot$ by SIAM. Unauthorized reproduction of this article is prohibited. 
From this expression of the adjoint operator, one can show that $\mathcal{A}^{*} g=\lambda g$ if and only if

$$
g=\left(\left(\lambda \mathrm{e}^{-\lambda \theta} I-A_{2}^{*}(\theta)+\lambda \mathrm{e}^{-\lambda \theta} \int_{0}^{\theta} \mathrm{e}^{\lambda s} A_{2}^{*}(s) \mathrm{d} s+\mathrm{e}^{-\lambda \theta} \int_{0}^{\theta} \mathrm{e}^{\lambda s} A_{3}^{*}(s) \mathrm{d} s\right) y\right),
$$

where $y \in \operatorname{Ker} \Delta^{*}(\lambda)$. Since $\mathcal{B}^{*} g=B^{*} y$, the proof of the lemma is complete.

Proof of Theorem 5.2. Let (i) be false. Then by Lemma 5.3 there exists a vector $g \neq 0$ such that $\mathcal{A}^{*} g=\lambda g$ and $g \in \operatorname{Ker} \mathcal{B}^{*}$. Consider an arbitrary state $\left(\begin{array}{c}y_{T} \\ z_{T}(\cdot)\end{array}\right) \in \mathcal{R}_{T}$, i.e., which is of the form (1.4). This gives

$$
\left\langle\left(\begin{array}{c}
y_{T} \\
z_{T}(\cdot)
\end{array}\right), g\right\rangle=\int_{0}^{T}\left\langle u(t), \mathcal{B}^{*} \mathrm{e}^{\mathcal{A}^{*} t} g\right\rangle \mathrm{d} t=0 .
$$

This means that $\mathcal{R}_{T}$ is not dense in $M_{2}$ and so cannot be equal to $\mathcal{D}(\mathcal{A})$ which is dense in $M_{2}$ because $\mathcal{A}$ is an infinitesimal generator. Hence null-controllability is impossible.

Now let condition (ii) not hold, i.e., there exists a nonzero vector $y \in \mathbb{C}^{n}$ such that

$$
A_{-1}^{*} y=\bar{\mu}_{m} y \quad \text { and } \quad B^{*} y=0 .
$$

With our notations, we can represent $y$ as

$$
y=\sum_{j=1}^{\nu_{m}} \alpha_{j} C_{m, j}^{1}
$$

where $C_{m, j}^{1}$ is a basis of the eigenspace of $A_{-1}^{*}$ corresponding to the eigenvalue $\bar{\mu}_{m}$. Among the moment equalities (3.16) we can extract those corresponding to $s=p_{m, j}$ (for fixed $m$ and $j=1, \ldots, \nu_{m}$ ), i.e.,

$$
s_{k}^{j}=k\left\langle\left(\begin{array}{c}
y_{T} \\
z_{T}(\cdot)
\end{array}\right), \psi_{m, k}^{j, p_{m, j}}\right\rangle=\sum_{d=1}^{r} \int_{0}^{T}\left(\mathrm{e}^{\lambda_{m}^{(k)} t} q_{m, k}^{j, p_{m, j}, d}(t)+f_{m, k}^{j, p_{m, j}, d}(t)\right) u_{d}(t) \mathrm{d} t
$$

for $|k|>N, j=1, \ldots, \nu_{m}$. From (3.14) and Lemma 3.2 we have

$$
q_{m, k}^{j, p_{m, j}, d}(t)=k\left\langle\mathbf{b}_{d}, \bar{\psi}_{m, k}^{j, p_{m, j}}\right\rangle=\frac{k}{\bar{\lambda}_{m}^{(k)}}\left\langle b_{d}, C_{m, j}^{1}\right\rangle
$$

Let us show that the moment problem (5.6) cannot be solved for all $\left\{s_{k}^{j}\right\} \in \ell_{2}$.

Assume the opposite; then the problem

$$
\widetilde{s}_{k}^{j}=\sum_{j=1}^{\nu_{m}} \bar{\alpha}_{j} s_{k}^{j}=\sum_{d=1}^{r} \int_{0}^{T} \sum_{j=1}^{\nu_{m}} \bar{\alpha}_{j}\left(\mathrm{e}^{\lambda_{m}^{(k)} t} q_{m, k}^{j, p_{m, j}, d}(t)+f_{m, k}^{j, p_{m, j}, d}(t)\right) u_{d}(t) \mathrm{d} t
$$

is also solvable for all $\left\{\widetilde{s}_{k}^{j}\right\} \in \ell_{2}$. On the other hand, (5.5) and (5.7) imply

$$
\sum_{j=1}^{\nu_{m}} \bar{\alpha}_{j} \mathrm{e}^{\lambda_{m}^{(k)} t} q_{m, k}^{j, p_{m, j}, d}(t)=\mathrm{e}^{\lambda_{m}^{(k)} t} \frac{k}{\bar{\lambda}_{m}^{(k)}}\left\langle b_{d}, \sum_{j=1}^{\nu_{m}} \alpha_{j} C_{m, j}^{1}\right\rangle=0 .
$$

Copyright $\odot$ by SIAM. Unauthorized reproduction of this article is prohibited. 
Hence the latter moment problem reads as

$$
\widetilde{s}_{k}^{j}=\sum_{d=1}^{r} \int_{0}^{T} g_{k}^{d}(t) u_{d}(t) \mathrm{d} t
$$

where $g_{k}^{d}(t)=\sum_{j=1}^{\nu_{m}} \bar{\alpha}_{j} f_{m, k}^{j, p_{m, j}, d}(t),|k|>N$, and, due to the property (P3), these functions satisfy

$$
\sum_{|k|>N} \int_{0}^{T}\left|g_{k}^{d}(t)\right|^{2} \mathrm{~d} t<\infty
$$

However, by Proposition 4.3 it follows that the set of solvability of (5.8) is a linear submanifold $\ell^{\prime} \subset \ell_{2}, \ell^{\prime} \neq \ell_{2}$. From the obtained contradiction, we conclude that

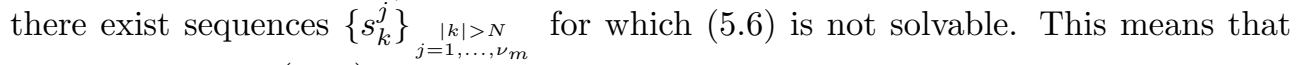
there exist states $\left(\begin{array}{c}y_{T}(\cdot) \\ z_{T}(\cdot)\end{array}\right)$ that satisfy $(\mathrm{C} 1)$ but are not reachable from 0 by the system (1.3). Thus $\mathcal{R}_{T} \neq \mathcal{D}(\mathcal{A})$.

The following results will be used to prove the main results on controllability. They are also of independent interest.

LEMma 5.4. Assume that for an abstract system (1.1) the following conditions hold:

(a) $\mathcal{R}_{T} \subset \mathcal{D}(\mathcal{A})$ for all $T>0$,

(b) for some $T_{0}>0$ the set $\mathcal{R}_{T_{0}}$ is a closed subspace of finite codimension in the space $X_{\mathcal{A}}=\mathcal{D}(\mathcal{A})$, with the standard graph norm $\|x\|_{\mathcal{A}}=\sqrt{\|x\|^{2}+\|\mathcal{A} x\|^{2}}$.

Then for all $T \geq T_{0}$ we have $\mathcal{R}_{T}=L$, where $L$ is a subspace of $\mathcal{D}(\mathcal{A})$ invariant by the semigroup $\mathrm{e}^{A t}$ and $0<\operatorname{codim} L \leq \operatorname{codim} \mathcal{R}_{T_{0}}<\infty$.

Proof. Taking into account the inclusion $\mathcal{R}_{T_{1}} \subset \mathcal{R}_{T_{2}}$ as $T_{1} \subset T_{2}$ we infer from assumptions (a) and (b) that there exists $\varepsilon>0$ such that

$$
\left.\left.\mathcal{R}_{T}=L, \quad T \in\right] T_{0}, T_{0}+\varepsilon\right],
$$

where $L$ is a subspace such that $0 \leq \operatorname{codim} L \leq \operatorname{codim} \mathcal{R}_{T_{0}}$. Let us show that the relation (5.9) holds also for all $T>T_{0}$. To do that it is enough to prove that

$$
\mathcal{R}_{T_{0}+\frac{3}{2} \varepsilon}=L
$$

Let us put

$$
\left.\mathcal{R}_{T_{2}}^{T_{1}}=\left\{x: x=\int_{T_{1}}^{T_{2}} \mathrm{e}^{\mathcal{A} t} B u(t) \mathrm{d} t, u \in L_{(} T_{1}, T_{2} ; U\right)\right\}
$$

and $\mathcal{R}_{T}^{0}=\mathcal{R}_{T}$. Let us prove first that

$$
\mathcal{R}_{T_{0}+\frac{3}{2} \varepsilon}^{T_{0}+\varepsilon} \subset L
$$

In fact, it is easy to see that $\mathcal{R}_{T_{0}+\frac{3}{2} \varepsilon}^{T_{0}+\varepsilon}=\mathrm{e}^{\mathcal{A} \frac{\varepsilon}{2}} \mathcal{R}_{T_{0}+\varepsilon}^{T_{0}+\frac{\varepsilon}{2}}$. On the other hand, it follows from (5.9) that $L=\mathcal{R}_{T_{0}+\varepsilon}=\mathcal{R}_{T_{0}+\frac{\varepsilon}{2}}$ and hence $\mathcal{R}_{T_{0}+\varepsilon}^{T_{0}+\frac{\varepsilon}{2}} \subset \mathcal{R}_{T_{0}+\varepsilon}=L$. Therefore

$$
\mathrm{e}^{\mathcal{A} \frac{\varepsilon}{2}} \mathcal{R}_{T_{0}+\varepsilon}^{T_{0}+\frac{\varepsilon}{2}} \subset \mathrm{e}^{\mathcal{A} \frac{\varepsilon}{2}} L=\mathrm{e}^{\mathcal{A} \frac{\varepsilon}{2}} \mathcal{R}_{T_{0}+\frac{\epsilon}{2}}=\mathcal{R}_{T_{0}+\varepsilon}^{\frac{\varepsilon}{2}} \subset \mathcal{R}_{T_{0}+\varepsilon}=L .
$$

Copyright (C) by SIAM. Unauthorized reproduction of this article is prohibited. 
Now from (5.11) and from the obvious relation

$$
\mathcal{R}_{T_{0}+\frac{3}{2} \varepsilon}=\mathcal{R}_{T_{0}+\varepsilon}+\mathcal{R}_{T_{0}+\frac{3}{2} \varepsilon}^{T_{0} \varepsilon}
$$

we infer that

$$
L \subset \mathcal{R}_{T_{0}+\frac{3}{2} \varepsilon}=\mathcal{R}_{T_{0}+\varepsilon}+\mathcal{R}_{T_{0}+\frac{3}{2} \varepsilon}^{T_{0}+\varepsilon} \subset L+L=L,
$$

which proves (5.10).

Thus (5.9) is valid for all $T>T_{0}$. Then $L=\cup_{T>0} \mathcal{R}_{T}$, and, therefore, it is an invariant subspace for the semigroup $\left\{\mathrm{e}^{\mathcal{A} t}\right\}_{t \geq 0}$. The lemma is proved.

In the following, we denote by $X_{\mathcal{A}}$ the space $\mathcal{D}(\mathcal{A}) \subset M_{2}$ with the graph norm.

THEOREM 5.5. For the system (1.3) let there exist a natural $N$ and $T_{0}>0$ such that the moment problem (3.16) for $T=T_{0}$ and $|k|>N$ is solvable for all the vectors

$$
\left\{k\left\langle\left(\begin{array}{c}
y_{T} \\
z_{T}(\cdot)
\end{array}\right), \psi_{m, k}^{j, s}\right\rangle\right\}_{|k|>N}
$$

satisfying the condition $(\mathrm{C} 1)$. Then, from the condition ( $\left.\mathrm{i}^{\prime}\right)$ of Lemma 5.3, it follows that $\mathcal{R}_{T}=\mathcal{D}(\mathcal{A})$ as $T>T_{0}$.

Proof. Let us denote by $L_{N} \subset \mathcal{D}(\mathcal{A})$ the subspace

$$
L_{N}=\mathrm{Cl}_{X_{\mathcal{A}}} \sum_{\substack{|k|>N \\ m=1, \ldots, \ell}} V_{m}^{(k)}
$$

and by $P_{N}$ a projector onto $L_{N}$ in $X_{\mathcal{A}}$. From the assumption on solvability of the problem (3.16), it follows that $P_{N} \mathcal{R}_{T_{0}}=L_{N}$. This, in particular, means that $\mathcal{R}_{T_{0}}$ is a subspace of finite codimension: $\operatorname{codim} L_{N}=(2 N+1) n$ in $\mathcal{D}(\mathcal{A})$. Then by Lemma 5.4 we conclude that $\mathcal{R}_{T}=L$ as $T>T_{0}$, where $L \subset \mathcal{D}(\mathcal{A})$ is invariant with respect to $\left\{\mathrm{e}^{\mathcal{A} t}\right\}_{t \geq 0}$ and $\operatorname{codim} L \leq(2 N+1) n$. Let us prove that under the condition (i') we have in fact $L=\mathcal{D}(\mathcal{A})$.

Assume the contrary. Then let us consider the dual space $X_{\mathcal{A}}^{*}$ and denote by $L^{\perp} \subset X_{\mathcal{A}}^{*}$ the subspace of functionals on $X_{\mathcal{A}}$ which are 0 on $L$. Obviously $L^{\perp}$ is finite dimensional. Denote by $\mathcal{A}_{1}^{*}$ the infinitesimal extension of $\mathcal{A}^{*}$ to the space $X_{\mathcal{A}}^{*}$ generating the semigroup $\mathrm{e}^{\mathcal{A}_{1}^{*} t}$. Since, due to Lemma 5.4, $L$ is invariant with respect to $\left\{\mathrm{e}^{\mathcal{A} t}\right\}_{t \geq 0}$, and then $L^{\perp}$ is invariant with respect to $\left\{\mathrm{e}^{\mathcal{A}_{1}^{*} t}\right\}_{t \geq 0}$. Taking into account the finite dimensionality of $L^{\perp}$, we conclude that $L^{\perp} \subset \mathcal{D}\left(\mathcal{\mathcal { A }}_{1}^{*}\right)$ and there exists an eigenvector $g$ of the operator $\mathcal{A}_{1}^{*}$ that lies in $L^{\perp}$. Let us notice now that the collection of subspaces $\left\{V_{m}^{(k)}, m=1, \ldots, \ell ;|k|>N\right\}$ is a Riesz basis also for the space $X_{\mathcal{A}}$ and all these subspaces are invariant for the operator $\mathcal{A}_{1}=\mathcal{A}_{\left.\right|_{\mathcal{D}(\mathcal{A})}}$. This implies that the collection $\left\{W_{m}^{(k)}, m=1, \ldots, \ell ;|k|>N ; \widehat{W}_{N}\right\}$ is a Riesz basis of invariant subspaces for $\mathcal{A}_{1}^{*}$ in the space $X_{\mathcal{A}}^{*}$. From this, we infer that all the eigenvectors of $\mathcal{A}_{1}^{*}$ lie in

$$
\bigcup_{m, k} W_{m}^{(k)} \bigcup \widehat{W}_{N} \subset \mathcal{D}\left(\mathcal{A}^{*}\right)
$$

and, therefore, $g$ is also an eigenvector for $\mathcal{A}^{*}$. Since $g \in L^{\perp}$, then

$$
\left\langle\int_{0}^{T} \mathrm{e}^{\mathcal{A} t} \mathcal{B} u(t) \mathrm{d} t, g\right\rangle=0, \quad u(\cdot) \in L_{2}(0, T ; U) .
$$

Copyright $@$ by SIAM. Unauthorized reproduction of this article is prohibited. 
If we put $u(t) \equiv u \in U, t \in[0, T]$, the latter relation brings

$$
0=\int_{0}^{T}\left\langle u, \mathcal{B}^{*} \mathrm{e}^{\mathcal{A}^{*} t} g \mathrm{~d} t\right\rangle=\int_{0}^{T}\left\langle u, \mathcal{B}^{*} g\right\rangle \mathrm{e}^{\mu t} \mathrm{~d} t=\left\langle u, \mathcal{B}^{*} g\right\rangle \int_{0}^{T} \mathrm{e}^{\mu t} \mathrm{~d} t
$$

for all $u \in U$, where $\mathcal{A}^{*} g=\bar{\mu} g$. This implies $g \in \operatorname{Ker} \mathcal{B}^{*}$, which contradicts $(\mathrm{C} 1)$. That completes the proof.

Now we are ready to prove the first important result of our work.

TheOREM 5.6. Let conditions (i) and (ii) of Theorem 5.2 hold. Then

(i) the system (1.3) is null-controllable at the time $T$ as $T>n$;

(ii) if the system (1.3) is of single control $(r=1)$, then the estimation of the time of controllability in (i) is exact, i.e., the system is not controllable at time $T=n$.

If the delay is $h$ instead of 1 , the time of exact controllability is $T=n h$.

Proof. Here we prove (i) for the case of a single control. In the case of multivariable control we obtain a more precise estimate for the time of controllability in section 6 .

First of all, let us observe that conditions (i) and (ii) of Theorem 5.2 imply, in the case of single control, that all the eigenspaces of $\mathcal{A}^{*}$ and $\widetilde{\mathcal{A}}^{*}$ are one dimensional. In fact, otherwise we will have that there exists an eigenvector $g$ of $\mathcal{A}^{*}$ or $\widetilde{\mathcal{A}}^{*}$ such that $\langle\mathbf{b}, g\rangle=0$. But we know that $g$ has the form

$$
g=\left(\begin{array}{c}
y \\
z(\theta)
\end{array}\right)
$$

where $y$ is a nonzero eigenvector for the pencil $\Delta^{*}(\lambda)$ (that is, $\Delta^{*}\left(\lambda_{0}\right) y=0$ for some $\left.\lambda_{0}\right)$ or of the matrix $A_{-1}^{*}$, respectively. Since $\langle\mathbf{b}, g\rangle=0$ gives $\langle b, y\rangle=0$ we arrive at a contradiction with the conditions of Theorem 5.2.

Thus, equalities (3.16) and (3.17) take, in our case, the form

$$
k\left\langle\left(\begin{array}{c}
y_{T} \\
z_{T}(\cdot)
\end{array}\right), \psi_{m, k}^{1, s}\right\rangle=\int_{0}^{T}\left(\mathrm{e}^{\lambda_{m}^{(k)} t} q_{m, k}^{1, s}(t)+f_{m, k}^{1, s}(t)\right) u(t) \mathrm{d} t
$$

where $|k|>N, m=1, \ldots, \ell, s=1, \ldots, p_{m, 1}$, and

$$
\left\langle\left(\begin{array}{c}
y_{T} \\
z_{T}(\cdot)
\end{array}\right), \widehat{\psi}_{m}^{1, s}\right\rangle=\int_{0}^{T} \mathrm{e}^{\widehat{\lambda}_{m} t} \widehat{q}_{m}^{1, s}(t) u(t) \mathrm{d} t
$$

where $m=1, \ldots, \ell_{N}, s=1, \ldots, \widehat{p}_{m, 1}$. From Lemmas 3.2 and 5.3 , it follows that all polynomials $\{q(t)\},\{\widehat{q}(t)\}$ are nontrivial and $\operatorname{deg} q_{m, k}^{1, s}(t)=p_{m, 1}-s, \operatorname{deg} \widehat{q}_{m}^{1, s}(t)=$ $\widehat{p}_{m, 1}-s$. This gives

$$
\sum_{m=1}^{\ell} p_{m, 1}^{\prime}=\sum_{m=1}^{\ell}\left(\operatorname{deg} q_{m, k}^{1,1}+1\right)=\sum_{m=1}^{\ell} p_{m, 1}=n .
$$

Applying Theorem 4.5, we find that for a large enough $N$, the collection

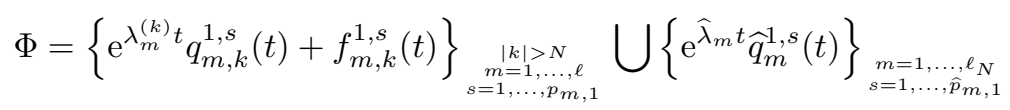

forms a Riesz basis in $\mathrm{Cl} \operatorname{Lin} \Phi \subset L_{2}(0, T)$. Then by Proposition 4.1 the moment problem (5.12) is solvable if and only if (C1) holds. Due to Theorem 5.5, this yields $\mathcal{R}_{T}=\mathcal{D}(\mathcal{A})$ for $T>n$. 
To prove (ii) we first recall that the total number of elements of the family

$$
\widehat{\Phi}=\left\{\mathrm{e}^{\widehat{\lambda}_{m} t} \widehat{q}_{m}^{1, s}(t), m=1, \ldots, \ell_{N} ; s=1, \ldots, \widehat{p}_{m, 1}\right\}
$$

equals $\sum_{m=1}^{\ell} \widehat{p}_{m, 1}=(2 N+2) n$. Since $\sum_{m=1}^{\ell} p_{m, 1}=n$, we have

$$
\sum_{m=1}^{\ell} \widehat{p}_{m, 1}=(2 N+1) \sum_{m=1}^{\ell} p_{m, 1}+n .
$$

On the other hand, it follows from Theorem 4.5 that in $L_{2}(0, n)$ we have

$$
\operatorname{codim} \mathrm{Cl} \operatorname{Lin} \Phi_{1}=(2 N+1) n=(2 N+1) \sum_{m}^{\ell} p_{m, 1},
$$

where

$$
\Phi_{1}=\left\{\mathrm{e}^{\lambda_{m}^{(k)} t} q_{m, k}^{1, s}(t)+f_{m, k}^{1, s}(t),|k|>N, m=1, \ldots, \ell, s=1, \ldots, p_{m, 1}\right\} .
$$

This means that the family $\Phi=\Phi_{1} \cup \widehat{\Phi}$ contains at least $n$ functions, which are presented as linear combinations of the others. As a consequence, the set of reachability $\mathcal{R}_{T}$ for $T=n$ cannot be equal to $\mathcal{D}(\mathcal{A})$. More precisely, the codimension of $\mathcal{R}_{T}$ in $\mathcal{D}(\mathcal{A})$ satisfies the estimation $n \leq \operatorname{codim} \mathcal{R}_{T}<\infty$. The theorem is proved.

Remark 5.7. It is clear that the system (1.3) is also uncontrollable at time $T<n$. Moreover, it follows from Proposition 4.2 that, in this case, the set $\mathrm{Cl} \mathcal{R}_{T}$ is of infinite codimension in $X_{\mathcal{A}}$.

6. Controllability in the multivariable case. Let us now consider the multivariable case: $\operatorname{dim} B=r$ with also the assumption that the matrix $A_{-1}$ is not singular, $\operatorname{det} A_{-1} \neq 0$.

Let $\left\{b_{1}, \ldots, b_{r}\right\}$ be an arbitrary basis noted $\beta$. Let us introduce a set of integers. We denote $B_{i}=\left(b_{i+1}, \ldots, b_{r}\right), i=0,1, \ldots, r-1$, which gives in particular $B_{0}=B$ and $B_{r-1}=\left(b_{r}\right)$ and we put formally $B_{r}=0$. We need in the following the integers $m_{i}^{\beta}=\operatorname{rank}\left(\begin{array}{llll}B_{i-1} & A_{-1} B_{i-1} & \cdots & A_{-1}^{n-1} B_{i-1}\end{array}\right)-\operatorname{rank}\left(\begin{array}{llll}B_{i} & A_{-1} B_{i} & \cdots & A_{-1}^{n-1} B_{i}\end{array}\right)$ $(6.1)$

corresponding to the basis $\beta$. Let us denote

$$
m_{1}=\max _{\beta} m_{1}^{\beta}, \quad \bar{m}=\min _{\beta} \max _{i} m_{i}^{\beta},
$$

for all possible choices of a basis $\beta$. It is easy to show that for all $\beta$, there exists $i$ such that $m_{i}^{\beta} \geq m_{1}$ and then $\bar{m} \geq m_{1}$. Indeed, assume that $m_{1}$ is realized on the basis $\beta=\left\{b_{1}, \ldots, b_{r}\right\}$, and consider an arbitrary basis $\beta_{0}=\left\{b_{1}^{0}, \ldots, b_{r}^{0}\right\}$. Then there exists $i$ such that $\operatorname{Lin}\left\{b_{i}^{0}, \ldots, b_{r}^{0}\right\} \not \subset \operatorname{Lin}\left\{b_{2}, \ldots, b_{r}\right\}$ but $\operatorname{Lin}\left\{b_{i+1}^{0}, \ldots, b_{r}^{0}\right\} \subset \operatorname{Lin}\left\{b_{2}, \ldots, b_{r}\right\}$. For this integer $i$ we have $m_{i}^{\beta_{0}} \geq m_{1}$.

Now we can formulate the main result of this section.

Theorem 6.1. Let conditions (i) and (ii) of Theorem 5.2 hold. Then

(i) the system (1.3) is null-controllable at the time $T>\bar{m}$;

(ii) the system (1.3) is not controllable at the time $T<m_{1}$. 
If the delay is $h$ instead of 1 , then in (i) and (ii) $\bar{m}$ and $m_{1}$ must be replaced by $\bar{m} h$ and $m_{1} h$, respectively.

Proof. We show first that the system is not controllable at the time $T<m_{1}$.

Assume that the system is controllable. Let the basis where $m_{1}$ is realized be $\left\{b_{1}, \ldots, b_{r}\right\}$. Consider now the relation (3.16) together with (3.14) and (3.15) given by the controllability problem. The basis $\{\widetilde{\psi}\}$ arising in (3.14) and (3.15) is given by (2.4) and expressed via the rootvectors $C_{m, j}^{s}$ of the matrix $A_{-1}^{*}$.

Let us choose the vectors $C_{m, j}^{s}$. Consider the subspace

$$
\operatorname{Im}\left(\begin{array}{llll}
B_{1} & A_{-1} B_{1} & \cdots & A_{-1}^{n-1} B_{1}
\end{array}\right),
$$

where $B_{1}=\left(\begin{array}{llll}b_{2} & b_{3} & \cdots & b_{r}\end{array}\right)$. Then the subspace

$$
\mathcal{N}_{1}=\left\{\operatorname{Im}\left(\begin{array}{llll}
B_{1} & A_{-1} B_{1} & \cdots & A_{-1}^{n-1} B_{1}
\end{array}\right)\right\}^{\perp}=\bigcap_{i=0}^{n-1} \operatorname{Ker} B_{1}^{*} A_{-1}^{*}{ }^{i}
$$

is invariant by $A_{-1}^{*}$. The condition of controllability gives that $A_{-\left.1\right|_{\mathcal{N}_{1}}}$ has, for each eigenvalue $\bar{\mu}$, only one Jordan chain. Indeed, on the contrary, if there are two chains in $\mathcal{N}_{1}$, then there are two independent eigenvectors corresponding to the same eigenvalue in $\mathcal{N}_{1} \subset \operatorname{Ker} B_{1}^{*}$, i.e., both these vectors are orthogonal to $b_{2}, \ldots, b_{r}$. Then there exists a linear combination of these eigenvectors, which is orthogonal also to $b_{1}$ and, as a consequence, belongs to $\operatorname{Ker} B^{*}$. This contradicts the controllability condition.

Note also that $\operatorname{dim} \mathcal{N}_{1}=n-\operatorname{dim} \operatorname{Im}\left(\begin{array}{llll}B_{1} & A_{-1} B_{1} & \cdots & A_{-1}^{n-1} B_{1}\end{array}\right)=m_{1}$. We take the corresponding vectors $C_{m, j}^{s}$ in $\mathcal{N}_{1}$. This implies

$$
\left\langle C_{m, j}^{s}, b_{i}\right\rangle=0, \quad i=2, \ldots, r .
$$

This means that $m$ is chosen such that $\bar{\mu}_{m}$ is an eigenvalue of $A_{-\left.1\right|_{\mathcal{N}_{1}}}^{*}, j$ is the number of the unique Jordan chain in $\mathcal{N}_{1}$, and $s$ is the index of the vectors in the Jordan chain.

Let $\Omega_{\mathcal{N}_{1}}$ be the set of indices of the eigenvalues $\bar{\mu}_{m} \in \sigma\left(A_{-\left.1\right|_{\mathcal{N}_{1}}}^{*}\right)$. For each $m \in \Omega_{\mathcal{N}_{1}}$ we have a Jordan chain, say with the number $j(m)$ in $\mathcal{N}_{1}$. The indices of the corresponding generalized eigenvectors are

$$
s \in\left\{p_{m, j(m)}, p_{m, j(m)}-1, \ldots, p_{m, j(m)}-p_{m, j(m)}^{\prime}+1\right\}=I_{m},
$$

the length of the Jordan chain is $p_{m, j(m)}^{\prime}$, and $C_{m, j(m)}^{p_{m, j(m)}}$ is an eigenvector. For $m \in$ $\Omega_{\mathcal{N}_{1}}, j(m)$ and $s \in I_{m}$ we consider the relation (3.16) which is the expression of the controllability condition. As $C_{m, j(m)}^{s} \in \mathcal{N}_{1}$, we get

$$
k\left\langle\left(\begin{array}{c}
y_{T} \\
z_{T}(\cdot)
\end{array}\right), \psi_{m, k}^{j(m), s}\right\rangle=\int_{0}^{T} \mathrm{e}^{\lambda_{m}^{(k)} t} q_{m, k}^{j(m), s, 1}(t) u_{1}(t) \mathrm{d} t+\sum_{d=1}^{r} \int_{0}^{T} f_{m, k}^{j, s, d}(t) u_{d}(t) \mathrm{d} t
$$

for $|k|>N$. From the hypothesis of controllability at the time $T$, it follows that the left-hand side gives an arbitrary element of $\ell_{2}$, and then the relation (6.3) may be represented by the expression

$$
x=\left[Q_{1}+F\right] u(\cdot), \quad x \in \ell_{2},
$$

Copyright ( $\odot$ by SIAM. Unauthorized reproduction of this article is prohibited. 
where the operators $Q_{1}$ and $F$ are linear bounded operators from $L_{2}(0, T)$ to $\ell_{2}$ defined by

$$
\begin{aligned}
Q_{1} u(\cdot) & =\left\{\int_{0}^{T} \mathrm{e}^{\lambda_{m}^{(k)} t} q_{m, k}^{j(m), s, 1}(t) u_{1}(t) \mathrm{d} t ; m \in \Omega_{\mathcal{N}_{1}},|k|>N, s \in I_{m}\right\}, \\
F u(\cdot) & =\left\{\sum_{d=1}^{r} \int_{0}^{T} f_{m, k}^{j(m), s, d}(t) u_{d}(t) \mathrm{d} t ; m \in \Omega_{\mathcal{N}_{1}},|k|>N, s \in I_{m}\right\} .
\end{aligned}
$$

We now need the following lemmas.

Lemma 6.2. The operator $F$ is compact.

Proof. By (P3) the operator $F$ is compact in the same way as $Q_{T}^{r}$ is compact in Proposition 4.3.

LEMma 6.3. The image of the operator $Q_{1}$ is of infinite codimension.

Proof. Let us recall that $m_{1}=\operatorname{dim} \mathcal{N}_{1}$. Then for each $k$, the sum of the length of Jordan chains of the operator $\widetilde{\mathcal{A}}$ corresponding to the Jordan chains in $\mathcal{N}_{1}$ is $m_{1}$ :

$$
\sum_{m \in \Omega_{N_{1}}} p_{m, j(m)}^{\prime}=m_{1}
$$

Let us first show that the family

$$
\left\{\mathrm{e}^{\lambda_{m}^{(k)} t} q_{m, k}^{j(m), s, 1}(t), m \in \Omega_{\mathcal{N}_{1}},|k|>N, s \in I_{m}\right\}
$$

forms a Riesz basis of the closure of its linear span in the space $L_{2}\left(0, m_{1}\right)$. In order to do that, we have to consider the family

$$
\left\{\mathrm{e}^{\lambda_{m}^{(k)} t}, \mathrm{e}^{\lambda_{m}^{(k)} t} t, \ldots, \mathrm{e}^{\lambda_{m}^{(k)} t} t^{p_{m, j(m)}-1} ; m \in \Omega_{\mathcal{N}_{1}},|k|>N,\right\} .
$$

This family forms a Riesz basis of the closure of its linear span in $L_{2}\left(0, m_{1}\right)$ by Theorem 4.4. Moreover, the closure of its linear span is of finite codimension $(2 N+1) m_{1}$ since it may be completed by a family of $(2 N+1) m_{1}$ functions to get a Riesz basis of $L_{2}\left(0, m_{1}\right)$. The relation between the families (6.5) and (6.6) may be written as

$$
\mathcal{T}\left(\mathrm{e}^{\lambda_{m}^{(k)}} t q_{m, k}^{j(m), s, 1}(t)\right)=\mathrm{e}^{\lambda_{m}^{(k)} t} t^{p_{m, j(m)}-s},
$$

where $\mathcal{T}$ is a linear bounded invertible operator in the closure of the linear span of the family (6.5). This implies that this family forms a Riesz basis in the closure of its linear span, which is of finite codimension. Then, from Proposition 4.2, the problem of moments

$$
s_{m, k}^{j(m), s}=\int_{0}^{T} \mathrm{e}^{\lambda_{m}^{(k)}} t q_{m, k}^{j(m), s, 1}(t) u_{1}(t) \mathrm{d} t
$$

is not solvable in a subspace of infinite codimension and this implies that $\operatorname{Im} Q_{1}$ is of infinite codimension. Lemma 6.3 is proved.

Let us now show that, from the fact that $\operatorname{Im} Q_{1}$ is of infinite codimension and $F$ is a compact operator, we have $\operatorname{Im}\left[Q_{1}+F\right] \neq \ell_{2}$.

The necessary and sufficient condition of the equality $\operatorname{Im}\left[Q_{1}+F\right]=\ell_{2}$ is (cf., for example, [13, Theorem 4.13])

$$
\exists \gamma>0 \quad \forall x \in \ell_{2}, \quad\left\|\left[Q_{1}+F\right]^{*} x\right\| \geq \gamma\|x\| .
$$

Copyright (c) by SIAM. Unauthorized reproduction of this article is prohibited. 
We know that $\operatorname{Ker} Q_{1}^{*}=\left(\operatorname{Im} Q_{1}\right)^{\perp}$ is an infinite-dimensional closed subspace. This implies that

$$
\forall x \in \operatorname{Ker} Q_{1}^{*}, \quad\left\|F^{*} x\right\| \geq \gamma\|x\|
$$

for the same scalar $\gamma$, and this is impossible because $F$ is a compact operator. Hence $\operatorname{Im}\left[Q_{1}+F\right] \neq \ell_{2}$ and this implies that (6.4) is not possible for all $x \in \ell_{2}$.

Then the relation $(6.3)$ is not possible for all $\left(y_{T}, z_{T}(\cdot)\right) \in \mathcal{D}(\mathcal{A})$ if $T<m_{1}$. Part (ii) of the theorem is proved.

Let us now prove part (i) of the theorem.

First we choose a basis for the relation (3.16). Let $\beta=\left\{b_{1}, \ldots, b_{r}\right\}$ be an arbitrary basis of $\operatorname{Im} B$ and $T>\max \left\{m_{i}^{\beta}, i=1, \ldots, r\right\}$. Consider now the subspaces

$$
\mathcal{N}_{i}=\bigcap_{j=0}^{n-1} \operatorname{Ker} B_{i}^{*} A_{-1}^{*}{ }^{j},
$$

where $B_{i}=\left(\begin{array}{lll}b_{i+1} & \cdots & b_{r}\end{array}\right), i=1, \ldots, r-1, B_{0}=B$, and $B_{r}=0$. We have $\mathcal{N}_{0}=0$, $\mathcal{N}_{r}=\mathbb{C}^{n}$, and $\mathcal{N}_{i} \subset \mathcal{N}_{i+1}$ for $i=0, \ldots, r-1$. The subspaces $\mathcal{N}_{i}$ are invariant by $A_{-1}^{*}$. In order to construct the basis $\{\psi\}$ corresponding to $|k|>N$, we first choose a basis of generalized eigenvectors of $A_{-1}^{*}$ in the following way. Let us take a basis in $\mathcal{N}_{1}$ as in the first part of the proof. Then we complete this basis up to a basis of $\mathcal{N}_{2}$ by extending some Jordan chains from $\mathcal{N}_{1}$ and by adding Jordan chains corresponding to some other eigenvalues. In the same way, we extend our basis up to the basis of $\mathcal{N}_{3}, \ldots, \mathcal{N}_{r}=\mathbb{C}^{n}$.

Remark 6.4. The part of the obtained basis of $\mathcal{N}_{i}$ not belonging to $\mathcal{N}_{i-1}, i=$ $1, \ldots, r$, does not contain two chains corresponding to the same eigenvalue. We have already proved that in $\mathcal{N}_{1}$ there do not exist two chains with the same eigenvalue. Suppose now that $\mathcal{N}_{2}$ contains the end of the first chain and the beginning of the second chain corresponding to the same eigenvalue. Let $y_{1}^{\circ} \in \mathcal{N}_{2}, y_{1}^{\circ} \notin \mathcal{N}_{1}$ be the continuation of the first chain from $\mathcal{N}_{1}$. If the maximal order of the rootvectors in $\mathcal{N}_{1}$ is $p$, then the order of $y_{1}^{\mathrm{o}}$ is $p+1$. Let $y_{2}^{\mathrm{n}}$ be a new eigenvector in the second chain of $\mathcal{N}_{2}$ corresponding to the same eigenvalue. Let us consider the vector $y=\alpha y_{1}^{\mathrm{o}}+\beta y_{2}^{\mathrm{n}}$. We know that $y_{1}^{\mathrm{o}}$ is not orthogonal to $b_{2}$, because if $y_{1}^{\mathrm{o}} \perp b_{2}$, then $y_{1}^{\mathrm{o}} \in \mathcal{N}_{1}$. Then one can choose $\alpha, \beta$ such that $y \perp b_{2}$. Then, for this choice of $\alpha$ and $\beta, y \in \mathcal{N}_{1}$ and it is a rootvector of higher order than $p$ in $\mathcal{N}_{1}$, which contradicts the construction of $\mathcal{N}_{1}$ (the maximal order in $\mathcal{N}_{1}$ is $p-1$ ). This proves the remark for $i=2$. For $i>2$, the proof is the same. of

We have then a basis in $\mathbb{C}^{n}$ of Jordan chains of $A_{-1}^{*}$ formed by successive bases

$$
\mathcal{N}_{1} \subset \mathcal{N}_{2} \subset \cdots \subset \mathcal{N}_{r}=\mathbb{C}^{n}
$$

Let us denote by $\Omega_{\mathcal{N}_{i} / \mathcal{N}_{i-1}}, i=1, \ldots, r\left(\Omega_{\mathcal{N}_{1} / \mathcal{N}_{0}}=\Omega_{\mathcal{N}_{1}}\right)$, the set of the indices $m$ of the eigenvalues of the matrix $A_{-1}^{*}$ for which there exists chains in $\mathcal{N}_{i}$ not belonging to $N_{i-1}$. Since for any $m \in \Omega_{\mathcal{N}_{i} / \mathcal{N}_{i-1}}$ such a chain is unique we can denote its number by $j(m)$.

Using the constructed basis, we obtain a basis $\{\psi\}$ by the relation (2.4). In this basis, the relations (3.16) may be written as follows, and noted as $\left(\mathbf{R}_{i}, i=1, \ldots, r\right)$. 
The first family $\left(\mathbf{R}_{1}\right)$ is

$$
\begin{aligned}
k\left\langle\left(\begin{array}{c}
y_{T} \\
z_{T}(\cdot)
\end{array}\right), \psi_{m, k}^{j(m), s}\right\rangle= & \int_{0}^{T} \mathrm{e}^{\lambda_{m}^{(k)} t} q_{m, k}^{j(m), s, 1}(t) u_{1}(t) \mathrm{d} t \\
& +\sum_{d=1}^{r} \int_{0}^{T} f_{m, k}^{j(m), s, d}(t) u_{d}(t) \mathrm{d} t
\end{aligned}
$$

for $m \in \Omega_{\mathcal{N}_{1}}, s=p_{m, j(m)}-p_{m, j(m)}^{1 \prime}+1, \ldots, \pi_{m, j(m)}^{1}, p_{m, j(m)}-1,|k|>N, \pi_{m, j(m)}^{1}=$ $p_{m, j(m)}$.

The second family $\left(\mathbf{R}_{2}\right)$ is

$$
\begin{aligned}
k\left\langle\left(\begin{array}{c}
y_{T} \\
z_{T}(\cdot)
\end{array}\right), \psi_{m, k}^{j(m), s}\right\rangle= & \int_{0}^{T} \mathrm{e}^{\lambda_{m}^{(k)} t} q_{m, k}^{j(m), s, 1}(t) u_{1}(t) \mathrm{d} t \\
& +\int_{0}^{T} \mathrm{e}^{\lambda_{m}^{(k)} t} q_{m, k}^{j(m), s, 2}(t) u_{2}(t) \mathrm{d} t \\
& +\sum_{d=1}^{r} \int_{0}^{T} f_{m, k}^{j(m), s, d}(t) u_{d}(t) \mathrm{d} t
\end{aligned}
$$

for $m \in \Omega_{\mathcal{N}_{2} / \mathcal{N}_{1}}, s=\pi_{m, j(m)}^{2}-p_{m, j(m)}^{2 \prime}+1, \ldots, \pi_{m, j(m)}^{2},|k|>N$, where $\pi_{m, j(m)}^{2}$ and $p_{m, j(m)}^{2 \prime}$ are some integer.

The last one $\left(\mathbf{R}_{r}\right)$ being

$$
\begin{aligned}
k\left\langle\left(\begin{array}{c}
y_{T} \\
z_{T}(\cdot)
\end{array}\right), \psi_{m, k}^{j(m), s}\right\rangle= & \sum_{d=1}^{r} \int_{0}^{T} \mathrm{e}^{\lambda_{m}^{(k)} t} q_{m, k}^{j(m), s, d}(t) u_{d}(t) \mathrm{d} t \\
& +\sum_{d=1}^{r} \int_{0}^{T} f_{m, k}^{j, s, d}(t) u_{d}(t) \mathrm{d} t
\end{aligned}
$$

for $m \in \Omega_{\mathcal{N}_{r} / \mathcal{N}_{r-1}}, j=j(m), s=\pi_{m, j(m)}^{r}-p_{m, j(m)}^{r \prime}+1, \ldots, \pi_{m, j(m)}^{r},|k|>N$, with some integer $\pi_{m, j(m)}^{r}$ and $p_{m, j(m)}^{r \prime}$.

For each $|k|>N$ the number of equalities $\left(\mathbf{R}_{i}, i=1, \ldots, r\right)$ is exactly $m_{i}^{\beta}$ (see the definition of this number in (6.1)).

Remark 6.5. Let us specify that in the collections $\left(\mathbf{R}_{i}, i=1, \ldots, r\right)$, for each $k$ there exists only one group of quasi polynomials corresponding to the given exponent. Moreover, for each $i, \ldots, r$, the quasi polynomials corresponding to $d=i$ have degrees $0,1, \ldots, p^{i \prime}$, as follows from $(2.4),(3.14)$, and Lemma 3.3.

Before solving the problems $\left(\mathbf{R}_{i}, i=1, \ldots, r\right)$ we solve first the same problems with $f_{m, k}^{j, s, d}=0$, noted $\left(\mathbf{R}_{i}^{0}, i=1, \ldots, r\right)$.

LEMma 6.6. The problems $\left(\mathbf{R}_{i}^{0}, i=1, \ldots, r\right)$ obtained from $\left(\mathbf{R}_{i}, i=1, \ldots, r\right)$ by the assumption that $f_{m, k}^{j, s, d}=0$ with $T>\max \left\{m_{i}^{\beta}, i=1, \ldots, r\right\}$ are solvable if and only if the left-hand side is an element of $\ell_{2}$.

Proof. Consider the problem $\left(\mathbf{R}_{1}^{0}\right)$ obtained from (6.7) with the assumption $f_{m, k}^{j, s, d}=0$. This problem is solvable if and only if the left-hand side is $\ell_{2}$ by Theorem 4.5. If this problem is not solvable, then the problems $\left(\mathbf{R}_{i}^{0}\right)$ are not solvable. If $\left(\mathbf{R}_{1}^{0}\right)$ is solvable, then we can find a solution $u_{1}(t)$. Then, in the problem $\left(\mathbf{R}_{2}^{0}\right)$ the term

$$
\int_{0}^{T} \mathrm{e}^{\lambda_{m}^{(k)} t} q_{m, k}^{j(m), s, 1}(t) u_{1}(t) \mathrm{d} t
$$

Copyright (c) by SIAM. Unauthorized reproduction of this article is prohibited. 
on the right-hand side is determined and may be moved to the left-hand side. Hence $\left(\mathbf{R}_{2}^{0}\right)$ is a new moment problem with unknown function $u_{2}(t)$. It is solvable if and only if the left-hand side is in $\ell_{2}$.

Repeating this argumentation up to $\left(\mathbf{R}_{r}^{0}\right)$, we obtain that the global problem $\left(\mathbf{R}_{i}^{0}, i=1, \ldots, r\right)$ is solvable if and only if the right-hand side in (6.7)-(6.9) is in $\ell_{2}$. The proof of Lemma 6.6 is complete.

Let us now return to the general problem $\left(\mathbf{R}_{i}, i=1, \ldots, r\right)$ given in (6.7)-(6.9). One can represent the equalities $\left(\mathbf{R}_{i}, i=1, \ldots, r\right)$ in the following operator form:

$$
x=Q_{N} u(\cdot)+F_{N} u(\cdot), \quad x \in \ell_{2}, \quad u(\cdot) \in L_{2}\left(0, T ; \mathbb{C}^{r}\right),
$$

with $N$ the integer for which the problem is considered $(|k|>N)$. We shall prove that there exists $N$ sufficiently large such that

$$
\operatorname{Im} Q_{N}=\ell_{2} \Longrightarrow \operatorname{Im}\left[Q_{N}+F_{N}\right]=\ell_{2},
$$

and the last equality means that $\left(\mathbf{R}_{i}, i=1, \ldots, r\right)$ is solvable if $\left(\mathbf{R}_{i}^{0}, i=1, \ldots, r\right)$ is solvable, i.e., if the left-hand sides in (6.7)-(6.9) are in $\ell_{2}$.

Suppose that $\operatorname{Im} Q_{N}=\ell_{2}$; then there exists a constant $\gamma_{N}>0$ such that $\left\|Q_{N}^{*} x\right\| \geq$ $\gamma_{N}\|x\|$ for all $x \in \ell_{2}$ (see, for example, [13, Theorem 4.13]). Let $N>N_{0}$ and let us denote by $\ell_{2}^{N}$ the Hilbert space $\ell_{2}(N)=\left\{s_{k},|k|>N: \sum_{|k| N}\left|s_{k}\right|^{2}<\infty\right\}$; then $Q_{N}=P Q_{N_{0}}$ where $P: \ell_{2}^{N_{0}} \rightarrow \ell_{2}^{N}$ is the projector defined by

$$
P\left(\left\{s_{k},|k|>N_{0}\right\}\right)=\left\{s_{k},|k|>N\right\} .
$$

Then $Q_{N}^{*}=Q_{N_{0}}^{*} P^{*}$ and $\left\|P^{*} x\right\|=\|x\|$. This gives

$$
\left\|Q_{N}^{*} x\right\|=\left\|Q_{N_{0}}^{*} P^{*} x\right\| \geq \gamma_{N_{0}}\|x\| .
$$

This means that for all $N>N_{0},\left\|Q_{N}^{*} x\right\| \geq \gamma\|x\|$ for all $x \in \ell_{2}$, where $\gamma=\gamma_{0}$.

Consider now the operator $F_{N}$. By the property (P3) (section 3) we have $\left\|F_{N}\right\| \rightarrow$ 0 when $N \rightarrow \infty$. Hence the norm $\left\|Q_{N}-Q_{N}-F_{N}\right\|=\left\|F_{N}\right\|$ can be made arbitrarily small, say $\left\|F_{N}\right\| \leq \frac{\gamma}{2}$. This gives that the operator $Q_{N}+F_{N}$ is also surjective because

$$
\left\|\left[Q_{N}^{*}+F_{N}^{*}\right] x\right\| \geq\left\|Q_{N}^{*} x\right\|-\left\|F_{N}^{*} x\right\| \geq \gamma\|x\|-\frac{\gamma}{2}\|x\|=\frac{\gamma}{2}\|x\| .
$$

Then from Lemma 6.6 it follows that if $T>\max \left\{m_{i}^{\beta}, i=1, \ldots, r\right\}$, the moment problem $\left(\mathbf{R}_{i}, i=1, \ldots, r\right),|k|>N$, is solvable for all left-hand sides in $\ell_{2}$.

Applying now Theorem 5.5, we conclude that $\mathcal{R}_{T}=\mathcal{D}(\mathcal{A})$. The proof of the theorem is complete.

7. Controllability in the general case. In the previous section, we use the assumption that the system (1.2) is a pure neutral-type system ( $\left.\operatorname{det} A_{-1} \neq 0\right)$. However, this condition is in fact a technical assumption that allows the use of the Riesz basis of eigenspaces of the operator $\mathcal{A}$ in $M_{2}$ and the moment problem approach.

In this section, we show that conditions (i) and (ii) of Theorem 5.2 are necessary and sufficient for exact controllability for the general neutral systems $\left(A_{-1}\right.$ may be a singular matrix). We obtain also the precise time of controllability. From Theorem 6.1 it is not clear what happens if the time $T$ is such that $m_{1} \leq T \leq \bar{m}$ even if the conditions of controllability are satisfied. In this section, the exact time of controllability is given. In order to do that, we need the classical concept of the controllability indices. 
Recall that the first index $n_{1}$ may be defined as the minimal integer $\nu$ such that (see, for example, [15, Chapter 5])

$$
\operatorname{rank}\left(B, A_{-1} B, \ldots, A_{-1}{ }^{\nu-1} B\right)=n .
$$

Lemma 7.1. Assume that the pair $\left(\mathcal{A}_{-1}, B\right)$ is controllable. Let $n_{1}$ be the index of controllability of the couple $\left(A_{-1}, B\right)$ and let $\bar{m}, m_{1}$ be defined by (6.2). Then $m_{1} \leq n_{1} \leq \bar{m}$.

Proof. Let $\beta=\left\{b_{1}, \ldots, b_{r}\right\}$ be an arbitrary basis of $\operatorname{Im} B$. Then

$$
A^{n_{1}} b_{1} \in \operatorname{Im}\left(\begin{array}{llll}
B & A B & \cdots & A^{n_{1}-1} B
\end{array}\right)=\operatorname{Im}\left(\begin{array}{llll}
B & A B & \cdots & A^{n-1} B
\end{array}\right) .
$$

This may be written as

$$
A^{n_{1}} b_{1} \in \operatorname{Lin}\left\{b_{1}, A b_{1}, \ldots, A^{n_{1}-1} b_{1}\right\}+\operatorname{Im}\left(\begin{array}{llll}
B_{1} & A B_{1} & \cdots & A^{n_{1}-1} B_{1}
\end{array}\right) .
$$

This gives that $m_{1}^{\beta} \leq n_{1}$ for all $\beta$. Hence $m_{1} \leq n_{1}$.

Let us now consider the indices $m_{1}^{\beta}, \cdots, m_{r}^{\beta}$. By the definition of the integers $m_{i}^{\beta}$ we get that

$$
\left\{b_{1}, \ldots, A^{m_{1}{ }^{\beta}-1} b_{1}, b_{2}, \ldots, A^{m_{2}{ }^{\beta}-1} b_{2}, \ldots, b_{r}, \ldots, A^{m_{r}{ }^{\beta}-1} b_{r}\right\}
$$

is a basis in $\mathbb{C}^{n}$. This may be verified remarking first that, by definition, the vectors $b_{r}, \ldots, A^{m_{r}^{\beta}-1} b_{r}$ are linearly independent and

$$
\operatorname{Lin}\left\{b_{r}, \ldots, A^{m_{r}^{\beta}-1} b_{r}\right\}=\operatorname{Lin}\left\{b_{r}, \ldots, A^{n-1} b_{r}\right\} .
$$

Then we have to consider the previous step, i.e., $m_{r-1}^{\beta}$, and state that

$$
\left\{b_{r-1}, \ldots, A^{m_{r-1}^{\beta}} b_{r-1}, b_{r}, \ldots, A^{m_{r}-1} b_{r}\right\}
$$

are also linearly independent and

$$
\begin{aligned}
\operatorname{Lin}\left\{b_{r-1}, \ldots, A^{m_{r-1}^{\beta}-1} b_{r-1}, b_{r}, \ldots, A^{m_{r}{ }^{\beta}-1} b_{r}\right\} \\
\quad=\operatorname{Lin}\left\{b_{r-1}, \ldots, A^{n-1} b_{r-1}, b_{r}, \ldots, A^{n-1} b_{r}\right\}
\end{aligned}
$$

and so on.

We have then $m_{1}^{\beta}+\cdots+m_{r}^{\beta}=n$ and then $\operatorname{rank}\left(\begin{array}{llll}B & A B & \cdots & A^{\bar{m}^{\beta}-1} B\end{array}\right)=n$, where $\max \left\{m_{i}^{\beta}, i=1, \ldots, r\right\}$. This gives $n_{1} \leq \bar{m}^{\beta}$ for all $\beta$ and hence $n_{1} \leq \bar{m}$. This completes the proof of the lemma.

It is well known that in contrast to indices $m_{1}, \bar{m}$, the controllability index $n_{1}$ is invariant under feedback. This means that $n_{1}$ is the same for all couples $\left(A_{-1}+\right.$ $B P, B)$, where $P$ is an $r \times n$ matrix. Then one can choose a feedback matrix $P$ and a basis in $\mathbb{C}^{n}$ such that $A_{-1}+B P$ take the following form (see [15, Theorem 5.10 and Corollary 5.3]):

$$
F=\operatorname{diag}\left\{F_{1}, \ldots, F_{r}\right\}
$$

where

$$
F_{i}=\left(\begin{array}{rrrrr}
0 & 1 & 0 & \cdots & 0 \\
0 & 0 & 1 & \cdots & 0 \\
\vdots & \vdots & \vdots & \ddots & \vdots \\
0 & 0 & 0 & \cdots & 1 \\
a_{1}^{i} & a_{2}^{i} & a_{3}^{i} & \cdots & a_{n_{i}}^{i}
\end{array}\right)
$$

Copyright $@$ by SIAM. Unauthorized reproduction of this article is prohibited. 
and $B$ becomes

$$
G=\operatorname{diag}\left\{g_{1}, \ldots, g_{r}\right\}
$$

where $g_{i}=\left(\begin{array}{llll}0 & 0 & \cdots & 1\end{array}\right)^{\mathrm{T}}$, the dimension being $n_{i} \times 1$. It is easy to check that $\bar{m}(F, G)=m_{1}(F, G)=n_{1}$. Moreover, the spectrum of $F$ may be chosen arbitrarily by means of an appropriate choice of $P$.

Let us now return to the controllability problem for the system (1.2) (or equivalently (1.3)). We first give a preliminary result.

Lemma 7.2. The system (1.2) is exactly null-controllable at the time $T$ if and only if the perturbed system

$$
\dot{z}(t)=\left(A_{-1}+B P\right) \dot{z}(t-1)+\int_{-1}^{0} A_{2}(\theta) \dot{z}(t+\theta) \mathrm{d} \theta+\int_{-1}^{0} A_{3}(\theta) z(t+\theta) \mathrm{d} \theta+B u
$$

is exactly null-controllable at the same time $T$.

Proof. Obviously it is enough to prove one implication only. Assume that the system (1.2) is controllable at the time $T$. It means that for any function $f(t) \in$ $H^{1}\left(T-1, T ; \mathbb{C}^{n}\right)$ there exists a control $u(t) \in L_{2}\left(0, T ; \mathbb{C}^{n}\right)$ such that the solution of the equation

$$
\dot{z}(t)=A_{-1} \dot{z}(t-1)+\int_{-1}^{0} A_{2}(\theta) \dot{z}(t+\theta) \mathrm{d} \theta+\int_{-1}^{0} A_{3}(\theta) z(t+\theta) \mathrm{d} \theta+B u(t),
$$

with the initial condition $z(t)=0, t \in[-1,0]$, verifies $z(t)=f(t), t \in[T-1, T]$. Let us rewrite (7.2) in the form

$$
\dot{z}(t)=\left(A_{-1}+B P\right) \dot{z}(t-1)+\int_{-1}^{0} A_{2}(\theta) \dot{z}(t+\theta) \mathrm{d} \theta+\int_{-1}^{0} A_{3}(\theta) z(t+\theta) \mathrm{d} \theta+B v(t),
$$

where $v(t)=u(t)-P \dot{z}(t-1), t \in[0, T]$. Since $z(t-1) \in H^{1}\left([0, T] ; \mathbb{C}^{n}\right)$, then $v(t) \in L_{2}\left(0, T ; \mathbb{C}^{n}\right)$. Thus, the control $v(t)$ transfers the state $z(t)=0, t \in[-1,0]$, to the state $z(t)=f(t), t \in[T-1, T]$, by virtue of the perturbed system. This means that it is also controllable at the time $T$.

We have the following result, which concludes our considerations.

THEOREM 7.3. Let the neutral-type system (1.2) be in the general form, i.e., without the assumption $\operatorname{det} A_{1} \neq 0$. Conditions (i) and (ii) of Theorem 5.2 are necessary and sufficient for the exact controllability of the system. Under these conditions, the precise time of controllability is $T=n_{1}$. This means that the system is not controllable for $T \leq n_{1}$ and is controllable for $T>n_{1}$.

If the delay is $h$ instead of 1 , then the exact time of controllability is $n_{1} h$.

Proof. According to Theorem 5.2, the proof of necessity is needed for the case when $\operatorname{det} A_{1}=0$. Let us first show that condition (ii) holds. Assume that (ii) is not verified. Then there exist vectors $z_{0} \neq 0$ such that $A_{-1}^{*} z_{0}=\lambda_{0} z_{0}$ and $B^{*} z_{0}=0$. If for all such vectors $\lambda_{0} \neq 0$, then one can find $P_{0}$ such that $A_{-1}+B P_{0}$ is not singular. Then, according to Lemma 7.2, the perturbed system (7.1) with $P=P_{0}$ is exactly null-controllable. This gives that the pair $\left(A_{-1}+B P_{0}, B\right)$ is controllable, which contradicts the existence of such vectors $z_{0}$. 
Suppose that for some vector $z_{0} \neq 0$, we have $A_{-1}^{*} z_{0}=0$ and $B^{*} z_{0}=0$. Then, multiplying (1.2) by $z_{0}$ we get

$$
\left\langle\dot{z}(t), z_{0}\right\rangle=\left\langle A_{-1} \dot{z}(t-1)+\int_{-1}^{0} A_{2}(\theta) \dot{z}(t+\theta) \mathrm{d} \theta+\int_{-1}^{0} A_{3}(\theta) z(t+\theta) \mathrm{d} \theta+B u(t), z_{0}\right\rangle
$$

and the exact null-controllability definition means that this relation holds for an arbitrary function $\left\langle\dot{z}(t), z_{0}\right\rangle \in L_{2}(T-1, T)$. As $\left\langle A_{-1} \dot{z}(t-1), z_{0}\right\rangle=0$ and $\left\langle B u(t), z_{0}\right\rangle=0$, this gives, after a change of variables,

$$
\begin{aligned}
& \left\langle\dot{z}(t), z_{0}\right\rangle=\left\langle\int_{-1}^{0} A_{2}(\theta) \dot{z}(t+\theta) \mathrm{d} \theta+\int_{-1}^{0} A_{3}(\theta) z(t+\theta) \mathrm{d} \theta, z_{0}\right\rangle \\
& =\left\langle\int_{t-1}^{t} A_{2}(s-t) \dot{z}(s) \mathrm{d} s+\int_{t-1}^{t} A_{3}(s-t) z(s) \mathrm{d} s, z_{0}\right\rangle \\
& =\left(\begin{array}{ll}
K_{2}\left(z_{0}\right) & K_{3}\left(z_{0}\right)
\end{array}\right)\left(\begin{array}{c}
\dot{z}(\cdot) \\
z(\cdot)
\end{array}\right),
\end{aligned}
$$

where $K_{j}: L_{2}\left((T-2, T) ; \mathbb{C}^{n}\right) \longrightarrow L_{2}(T-1, T), j=2,3$, are linear operators defined by

$$
\left(K_{j}\left(z_{0}\right) w\right)(t)=\int_{t-1}^{t} A_{j}(s-t) w(s) \mathrm{d} s=\int_{T-1}^{T} \widehat{A}_{j}(s-t) w(s) \mathrm{d} s
$$

and

$$
\widehat{A}_{j}(s)=\left\{\begin{array}{cc}
A_{j}(s), & s \in[-1,0] \\
0, & s \notin[-1,0] .
\end{array}\right.
$$

The operators $K_{j}, j=2,3$, are clearly compact operators because

$$
\int_{T-1}^{T}\left(\int_{T-2}^{T}\left\|\widehat{A}_{j}(s-t)\right\|^{2} \mathrm{~d} s\right) \mathrm{d} t=\int_{T-1}^{T}\left(\int_{-1}^{0}\left\|A_{j}(\theta)\right\|^{2} \mathrm{~d} \theta\right) \mathrm{d} t<\infty ;
$$

see, for example, [7, Chapter 6]. Then the image of the operator $\left(K_{2}\left(z_{0}\right) \quad K_{3}\left(z_{0}\right)\right)$ cannot coincide with $L_{2}(T-1, T)$. Thus, such a vector $z_{0}$ does not exist. This gives that condition (ii) is necessary.

Let us now prove the necessity of condition (i). If $A_{-1}$ is nonsingular, it is proved in Theorem 5.2. Assume now that $A_{-1}$ is singular. Then (since we have proved (ii)) we can choose a matrix $P$ such that $A_{-1}+B P$ is not singular. According to Lemma 7.2, the perturbed system (7.1) is still exactly null-controllable. Using Theorem 5.2, we have the following statement: there do not exist $\lambda \in \mathbb{C}$ and $y \in \mathbb{C}^{n}$ such that

$$
\left[\lambda I-\lambda \mathrm{e}^{-\lambda}\left(A_{-1}^{*}+P^{*} B^{*}\right)-\lambda \int_{-1}^{0} \mathrm{e}^{\lambda s} A_{2}^{*}(s) \mathrm{d} s-\int_{-1}^{0} \mathrm{e}^{\lambda s} A_{3}^{*}(s) \mathrm{d} s\right] y=0
$$

and $B^{*} y=0$. This gives condition (i).

Now we prove the sufficiency. Assume that conditions (i) and (ii) are verified. Then they are also verified for the perturbed system. From condition (ii) we can choose a matrix $P$ such that $A_{-1}+B P$ is nonsingular and $\bar{m}\left(A_{-1}+B P, B\right)=m_{1}\left(A_{-1}+\right.$ $B P, B)=n_{1}$. And this gives that the perturbed system is exactly null-controllable at the time $T>n_{1}$ and is not controllable at the time $T<n_{1}$. By Lemma 7.2 we infer that our system (1.2) satisfies the same condition.

Copyright (c) by SIAM. Unauthorized reproduction of this article is prohibited. 
Moreover, it is easy to prove, arguing as in the proof of Theorem 5.6, that the system (1.2) is also not controllable at the time $T=n_{1}$. More precisely, the codimension of $\mathcal{R}_{n_{1}}$ in $X_{A}$ is finite and not less than $n_{1}$. For $T<n_{1}$, the codimension of $\mathcal{R}_{T}$ is infinite.

8. Conclusion and perspectives. The main goal of this paper is to demonstrate how the moment problem approach can be used in the controllability problem for delay systems of neutral type. To this end, we chose a quite general model (1.2) with distributed delays in the function and its derivative, a pointwise neutral term determined by a matrix $A_{-1}$, and the control term by a matrix $B$. Using our approach, we have given a complete analysis of the exact null-controllability for this model. Namely,

(i) we showed that the maximal possible set of the states reachable from 0 by the system at some time $T>0$ is the space $H^{1}$;

(ii) we found the conditions of the parameters of the system under which this set of reachability can be maximally possible (the conditions of exact controllability);

(iii) we proved that, under the above conditions, the system is exactly controllable at the time $T$ if and only if $T>n_{1}$, where $n_{1}$ is the first controllability index of the couple $\left(A_{-1}, B\right)$ (the time of exact controllability).

As a perspective, we consider the extension of our approach to systems with several pointwise neutral terms and to the general case of distributed neutral-type delay,

$$
K f=\int_{-1}^{0} \mathrm{~d} \mu(\theta) f(\theta), \quad f \in C\left([-1,0], \mathbb{C}^{n}\right),
$$

where $\mu$ is a matrix-valued function of bounded variation and continuous at zero. One can prove that, for this class of systems, the generalized Riesz basis property of the model operator $\mathcal{A}$ is preserved. However, the immediate spectral analysis of this operator is more complex. In the case when the delays in the neutral terms are commensurable, the results on exact controllability are expected to be similar to those obtained in the present paper. In the general case, the formulation and the proofs may be much more complicated. This problem is to be considered in our forthcoming works.

\section{REFERENCES}

[1] S. A. Avdonin And S. A. Ivanov, Families of Exponentials. The Method of Moments in Controllability Problems for Distributed Parameter Systems, Cambridge University Press, Cambridge, UK, 1995.

[2] H. T. Banks, M. Q. Jacobs, And C. E. Langenhop, Characterization of the controlled states in $W_{2}^{(1)}$ of linear hereditary systems, SIAM J. Control, 13 (1975), pp. 611-649.

[3] J. A. Burns, T. L. Herdman, and H. W. Stech, Linear functional differential equations as semigroups on product spaces, SIAM J. Math. Anal., 14 (1983), pp. 98-116.

[4] I. C. Gohberg And M. G. KRein, Introduction to the Theory of Linear Nonselfadjoint Operators, Transl. Math. Monogr. 18, AMS, Providence, RI, 1969.

[5] K. Ito and T. J. TARn, A linear quadratic optimal control for neutral systems, Nonlinear Anal., 9 (1985), pp. 699-727.

[6] M. Q. JaCobs AND C. E. LANGenhop, Criteria for function space controllability of linear neutral systems, SIAM J. Control Optim., 14 (1976), pp. 1009-1048.

[7] L. A. Liusternik and V. J. Sobolev, Elements of Functional Analysis, Russian Monographs and Texts on Advanced Mathematics and Physics, 5, Hindustan Publishing, Delhi, Gordon and Breach Publishers, New York, 1961. 
[8] A. Manitius and R. Triggiani, Function space controllability of linear retarded systems: A derivation from abstract operator conditions, SIAM J. Control Optim., 16 (1978), pp. 599645.

[9] D. A. O'Connor and T. J. TARn, On the function space controllability of linear neutral systems, SIAM J. Control Optim., 21 (1983), pp. 306-329.

[10] R. Rabah, G. M. Sklyar, and A. V. Rezounenko, Generalized Riesz basis property in the analysis of neutral type systems, C. R. Math. Acad. Sci. Paris, 337 (2003), pp. 19-24.

[11] R. Rabah, G. M. Sklyar, and A. V. Rezounenko, Stability analysis of neutral type systems in Hilbert space, J. Differential Equations, 214 (2005), pp. 391-428.

[12] H. Rivera Rodas and C. E. Langenhop, A sufficient condition for function space controllability of a linear neutral system, SIAM J. Control Optim., 16 (1978), pp. 429-435.

[13] W. Rudin, Functional Analysis, 2nd ed., McGraw-Hill, New York, 1991.

[14] D. Ullrich, Divided differences and systems of nonharmonic Fourier series, Proc. Amer. Math. Soc., 80 (1980), pp. 47-57.

[15] W. M. Wonham, Linear Multivariable Control: A Geometric Approach, Springer, New York, 1985.

[16] Y. Үамамото, Reachability of a class of infinite-dimensional systems: An external approach with applications to general neutral systems, SIAM J. Control Optim., 27 (1989), pp. 217234.

[17] R. Y. Young, An Introduction to Nonharmonic Analysis, Academic Press, New York, 1980.

Copyright (c) by SIAM. Unauthorized reproduction of this article is prohibited. 